\title{
Numerical simulations of the Lagrangian averaged Navier-Stokes equations for homogeneous isotropic turbulence
}

\author{
Kamran Mohseni ${ }^{\mathrm{a})}$ \\ Department of Aerospace Engineering Sciences, University of Colorado, Boulder, Colorado 80309-0429 \\ Branko Kosović and Steve Shkoller \\ Department of Mathematics, University of California, Davis, California 95616-8633 \\ Jerrold E. Marsden \\ Division of Engineering and Applied Science, California Institute of Technology, Pasadena, California 91125
}

(Received 8 November 2001; accepted 6 November 2002; published 8 January 2003)

\begin{abstract}
Capabilities for turbulence calculations of the Lagrangian averaged Navier-Stokes (LANS- $\alpha$ ) equations are investigated in decaying and statistically stationary three-dimensional homogeneous and isotropic turbulence. Results of the LANS- $\alpha$ computations are analyzed by comparison with direct numerical simulation (DNS) data and large eddy simulations. Two different decaying turbulence cases at moderate and high Reynolds numbers are studied. In statistically stationary turbulence two different forcing techniques are implemented to model the energetics of the energy-containing scales. The resolved flows are examined by comparison of the energy spectra of the LANS- $\alpha$ with the DNS computations. The energy transfer and the capability of the LANS- $\alpha$ equations in representing the backscatter of energy is analyzed by comparison with the DNS data. Furthermore, the correlation between the vorticity and the eigenvectors of the rate of the resolved strain tensor is studied. We find that the LANS- $\alpha$ equations capture the gross features of the flow, while the wave activity below the scale $\alpha$ is filtered by a nonlinear redistribution of energy.

(c) 2003 American Institute of Physics. [DOI: 10.1063/1.1533069]
\end{abstract}

\section{INTRODUCTION}

Over the last 30 years, direct numerical simulation of turbulent flows at small to moderate Reynolds numbers has been a valuable asset in understanding turbulence phenomena. In such simulations, the motion of eddies ranging in size from the physical system down to the Kolmogorov dissipation length scale are explicitly accounted for. The capability of reproducing experimentally obtained data for various flows has been demonstrated by these simulations; see Rogallo and Moin ${ }^{1}$ and Canuto et al. ${ }^{2}$ for an earlier account on the progress in this area. The main difficulty in the turbulence engineering community is that performing direct numerical simulations (DNS) of typical engineering problems (usually at high Reynolds numbers) is computationally very expensive, and therefore not likely to become feasible in the foreseeable future. This is mainly because the number of degrees of freedom for a three-dimensional Navier-Stokes flow grows rapidly with Reynolds number; namely it is proportional to $\mathrm{Re}^{9 / 4}$. Consequently, increasing the Reynolds number by a factor of 2 will increase the memory size by about a factor of 5 and the computational time by an order of magnitude. At low Reynolds numbers, DNS remains a unique computational tool that provides information about a number of quantities inaccessible in a laboratory; however, alternative approaches are needed for numerical simulations of high Reynolds number flows.

\footnotetext{
a) Telephone: (303) 492 0286; fax: (303) 492 7881; electronic mail: mohseni@colorado.edu
}

Alternative approaches to simulations of turbulent flows where one does not use the brute-force approach to resolve all important scales of motion like in DNS or for high Reynolds number flows for which DNS is not feasible are usually divided into two groups: Reynolds averaged NavierStokes (RANS) simulations and large eddy simulations (LES).

In the RANS approach, the flow field is decomposed into an ensemble averaged mean flow and a fluctuating perturbation field. By invoking the ergodic hypothesis, ensemble averages are usually approximated by time averages or averages over one or more spatial dimensions. Substituting this decomposition into the Navier-Stokes equations results in a set of differential equations for the mean flow quantities containing contributions from the time-varying, turbulent motion. This requires the introduction of a turbulence model to describe the effect of these fluctuations on the mean. However, by providing only mean turbulence quantities, the RANS approach significantly limits our ability to study spatio-temporal structures in turbulent flows.

This problem can be circumvented by using the LES approach. In LES, only large scales of motion are resolved while the effect of small scales is modeled. The basic idea behind LES is to define a large scale field through low-pass filtering of the flow variables. The governing equations for the mean flow quantities (large scales) are obtained by filtering the Navier-Stokes and continuity equations. In inhomogeneous, e.g., wall bounded flows, the filter width must be a function of position to capture the average size of the turbu- 
lent eddies that vary in space. Due to computational limitations of the DNS approach, the LES technique has matured into a commonly used tool for studying turbulent flows in engineering applications.

The closure problem in LES and RANS is a central issue in turbulence modeling. It is believed that turbulence at small scales retains a higher level of homogeneity, which makes it more susceptible to modeling. The rationale is that only the large scale motions are noticeably affected by the geometry of the domain, while the small scale motions are self-similar or even universal throughout the bulk of the flow. Hence, the main goal of LES modeling is to accurately model the net effect of small (subgrid) scales on the dynamics of large scales (grid scales) without solving for the evolution of small scales.

LES as a method for numerical simulations of threedimensional turbulent flows was developed by Lilly. ${ }^{3}$ Lilly's turbulence modeling approach was based on that used by Smagorinsky ${ }^{4}$ in which subgrid scale (SGS) stresses are linearly related to the rate of deformation tensor with the positive semi-definite subgrid eddy viscosity as a proportionality factor. The first simulations based on Lilly's model were performed by Deardorff ${ }^{5}$ who used LES to study high Reynolds number boundary layers, such as those occurring in the atmosphere. Smagorinsky-type models are dissipative and cannot represent normal stress effects in sheared turbulent flows. These limitations of linear, Smagorinsky-type models have been recognized after first LES were carried out. ${ }^{5,6}$ In an attempt to develop a better subgrid model, Leonard ${ }^{7}$ expanded the filtered nonlinear term as a function of products of filtered quantities and their derivatives and obtained a gradient diffusivity model. McMillan and Ferziger ${ }^{8}$ and Clark et $a l^{9}{ }^{9}$ devised an a priori testing approach where they filter DNS data to compute subgrid terms (using their definitions) and compare them to modeled subgrid terms. Bardina et al. ${ }^{10}$ used such tests to evaluate a scale similarity model that they developed. Scale similarity models are now often combined with Smagorinsky-type models and are commonly used in LES. $^{11}$

The dynamic approach to subgrid modeling was developed by Germano et al., ${ }^{12}$ who used an additional, test filter and tensor identities to dynamically determine the value of the Smagorinsky coefficient. Such dynamic subgrid models allow for negative eddy viscosity and can therefore represent the backscatter of energy from small, unresolved scales of motion toward large, resolved eddies. Following Rivlin's ${ }^{13}$ suggestion, a class of Reynolds and subgrid stress models was developed based (as discussed later) on the constitutive theory for second grade fluids. Due to their relation to the LANS- $\alpha$ subgrid terms, these models will be discussed in more detail in Sec. II. Subgrid parameterizations are necessarily based on simplifying assumptions and a phenomenological approach, and represent the main source of uncertainties and errors in LES. While some of the subgrid models perform satisfactory in specific flow configurations, a general subgrid modeling problem remains open. In addition to a recent review by Meneveau and $\mathrm{Katz}^{11}$ that focuses on scalesimilarity models detailed reviews of the LES technique have been presented by Mason ${ }^{14}$ and Lesieur and Métais. ${ }^{15}$
Recently, Hughes et al. ${ }^{16-18}$ described a two level variational multiscale method for large eddy simulation where they used a priori scale separation instead of commonly used spatial filtering. Collis ${ }^{19}$ extended this idea to a three-level approach to clarify the role of unresolved scales.

In this study, we consider a new Lagrangian averaging approach introduced in Holm et al. ${ }^{20}$ and Marsden and Shkoller. ${ }^{21}$ Unlike the traditional averaging or filtering approach used for both RANS and LES, wherein the NavierStokes equations are averaged, the Lagrangian averaging approach is based on averaging at the level of the variational principle from which the Navier-Stokes equations are derived. Namely, a new averaged action principle is defined. Hamilton's principle then yields the Lagrangian-averaged Euler (LAE- $\alpha$ ) equations when the flow is deterministic; when the flow is a stochastic process and covariant derivatives are replaced by mean backward-in-time stochastic derivatives, the Lagrangian-averaged Navier-Stokes equations (LANS- $\alpha$ ) are obtained, via the Ito formula of stochastic calculus (just as the Navier-Stokes equations are obtained from the usual non-averaged action principle). The Lagrangian averaging procedure yields the LANS- $\alpha$ equations that describe the time evolution of large eddies in turbulent flows. In this sense, the Lagrangian averaging approach is similar to that of LES.

The Lagrangian averaged Euler models were introduced on all of $\mathbb{R}^{3}$ (the three-dimensional Euclidean space) in Holm et al., ${ }^{22}$ on boundaryless manifolds in Shkoller, ${ }^{23}$ on bounded subsets of $\mathbb{R}^{3}$ with boundary in Marsden et al., ${ }^{24}$ and on manifolds with boundary in Shkoller; ${ }^{25}$ they were derived to model the mean motion of incompressible flows. A short review of the derivation of the Lagrangian-averaged equations is presented in Sec. II.

The behavior of small scales in turbulent flows is often characterized by statistical isotropy, homogeneity, and universality. Consequently, by investigating the simplest turbulent flow, i.e., isotropic homogeneous turbulence, we hope to understand small scale turbulence. In this study, we shall concentrate on homogeneous isotropic flows. Such flows are unbounded and thus differ from flow in regions near solid boundaries, but they provide an ideal test case for the adjustment and verification of new turbulence theories and models.

Isotropic homogeneous turbulence can be categorized as either decaying or forced. While the forced isotropic turbulence is usually designed to be statistically stationary, decaying turbulence is always statistically nonstationary. Recently, Chen et al. ${ }^{26}$ performed numerical computations of the forced LANS- $\alpha$ equations for homogeneous turbulence. They considered only one forcing technique, similar to the forcing scheme B of the present study (see Sec. V). In this study we perform similar computations, extend the results of Chen et al., ${ }^{26}$ and implement a different forcing scheme. ${ }^{27}$ However, to obtain a better understanding of the modeling capabilities of the Lagrangian averaging technique we consider more realistic idealizations of a turbulent flow than the forced homogeneous turbulence cases. Consequently in this study we consider two different decaying turbulent flows as well. In the first case an initial condition from a realization of the experiments by Comte-Bellot and Corrsin ${ }^{28,29}$ is used. ${ }^{30}$ 
This initial condition has a broad energy spectrum with the peak of the energy spectrum at a relatively high wave number. In the second case we generate a sharply peaked initial energy spectrum with the peak of the energy spectrum at a relatively low wave number. In this way the effects of the initial energy spectrum on the performance of the LANS- $\alpha$ calculations are also investigated.

This paper is organized as follows. In the next section a review of the Lagrangian averaging technique is presented. The numerical technique adopted in this study is described in Sec. III. Our main numerical results are presented in Secs. IV-VI where computations on the LAE- $\alpha$ as well as forced and decaying LANS- $\alpha$ calculations are discussed. Finally our findings are summarized in Sec. VII.

\section{THE LAGRANGIAN AVERAGING TECHNIQUE}

In this section, we give a brief summary of the relevant background material on the Lagrangian averaging approach and the resulting Lagrangian averaged Euler (LAE- $\alpha$ ) and Lagrangian-averaged Navier-Stokes (LANS- $\alpha$ ) equations. The detailed derivation of the LAE- $\alpha$ and LANS- $\alpha$ equations can be found in the article. ${ }^{31}$ Analytic results concerning these equations can be found in the literature. ${ }^{21,23-25,32-34}$

The well-known Reynolds decomposition is an additive decomposition of the spatial velocity field of the fluid into its mean and fluctuating components. The Lagrangian averaging procedure takes an entirely different starting point, by decomposing instead the Lagrangian flow of the velocity field.

Let $X$ denote the vector space of initial velocity fields for which the Euler equations are (at least locally) well-posed, and let $\Sigma$ denote the unit sphere in $X$. For $u_{0} \in X$, let $u(t, x)$ denote the solution of the Euler equations with $u(0, \cdot)=u_{0}$. Similarly, let $u^{\epsilon}(t, x)$ denote the solution of the Euler equations with initial data $u_{0}^{\epsilon}$, where

$$
u_{0}^{\epsilon}=u_{0}+\epsilon w, \quad w \in \Sigma, \quad \epsilon \in[0, \alpha]
$$

for some small $\alpha>0$. Of course, $u^{\epsilon}(t, x)$ depends on $w$ as well, but we suppress that for notational simplicity.

We let $\nu$ denote a chosen measure on the unit sphere $\Sigma$ in $X$, and define the average of vector-valued functions $f(\epsilon, w)$ on $[0, \alpha] \times \Sigma$ by

$$
\langle f\rangle=\frac{t}{\alpha} \int_{0}^{\alpha} \int_{\Sigma} f(\epsilon, w) \nu d \epsilon,
$$

where $t$ is a characteristic time scale.

Let $\eta$ be the Lagrangian flow of $u$, which solves $\left.\partial_{t} \eta(t, x)=u(t, \eta(t, x))\right)$ with $\eta(0, x)=x$. Similarly, let $\eta^{\epsilon}$ denote the Lagrangian flow of $u^{\epsilon}$. We define the Lagrangian fluctuation volume-preserving diffeomorphism $\xi^{\epsilon}$ by

$$
\xi^{\epsilon}(t, x)=\eta^{\epsilon}\left(t, \eta^{-1}(t, x)\right),
$$

so that $\eta_{t}^{\epsilon}=\xi_{t}^{\epsilon} \circ \eta_{t}$, with $\circ$ denoting composition of maps. Clearly, $\xi^{0}(t, x)=x$, since $\eta^{\epsilon=0}(t, x)=\eta(t, x)$ for all $t \geqslant 0$. Let

$$
u^{\prime}(t, x)=\left.\frac{d}{d \alpha}\right|_{\alpha=0} u^{\alpha}(t, x)
$$

denote the associated Eulerian velocity fluctuation about $u$. The corresponding Lagrangian fluctuation (in spatial representation) is given by

$$
\xi^{\prime}(t, x)=\left.\frac{d}{d \alpha}\right|_{\alpha=0} \xi^{\alpha}(t, x) .
$$

Similarly, let

$$
u^{\prime \prime}(t, x)=\left.\frac{d^{2}}{d^{2} \alpha}\right|_{\alpha=0} u^{\alpha}(t, x),
$$

and

$$
\xi^{\prime \prime}(t, x)=\left.\frac{d^{2}}{d^{2} \alpha}\right|_{\alpha=0} \xi^{\alpha}(t, x) .
$$

Recall that the inviscid portion of the dynamics of the Navier-Stokes equations is governed by a simple variational principle, or action, which is just the time integral of the kinetic energy of divergence-free vector fields:

$$
S=\frac{1}{2} \int_{t_{0}}^{t_{1}} \int_{\Omega}\left|\partial_{t} u^{\epsilon}(t, x)\right|^{2} d x d t .
$$

The Euler-Lagrange equations for $S(u)$ are the incompressible Euler equations, and if one allows the flow of the Euler solution to undergo a random walk, then the Navier-Stokes equations immediately arise. ${ }^{35,36}$ Our goal is to average over all possible solutions of the Euler equations with initial data $u_{0}^{\epsilon}$ in an $X$-ball of radius $\alpha$ about $u$; since each solution $u^{\epsilon}(t, x)$ is obtained from the first variation of the action as we described above, it is appropriate to define the averaged action function

$$
\bar{S}=\left\langle\frac{1}{2} \int_{0}^{T} \int_{\Omega}\left|\partial_{t} u^{\epsilon}\right|^{2} d x d t\right\rangle .
$$

Here, overbar denotes averaged quantity. Expanding $u^{\epsilon}$ about $\epsilon=0$, we get

$$
u^{\epsilon}(t, x)=u(t, x)+\epsilon u^{\prime}(t, x)+\frac{1}{2} \epsilon^{2} u^{\prime \prime}(t, x)+O\left(\epsilon^{3}\right) .
$$

We insist that our measure is invariant so that $\left\langle u^{\epsilon}\right\rangle=u$; correspondingly, we call $u$ the mean. By differentiating Eq. (1), one obtains the relations between Eulerian and Lagrangian fluctuations

$$
\begin{aligned}
u^{\prime}= & \partial_{t} \xi^{\prime}+(u \cdot \nabla) \xi^{\prime}-\left(\xi^{\prime} \cdot \nabla\right) u, \\
u^{\prime \prime}= & \partial_{t} \xi^{\prime \prime}+(u \cdot \nabla) \xi^{\prime \prime}-\left(\xi^{\prime \prime} \cdot \nabla\right) u-2\left(\xi^{\prime} \cdot \nabla\right) u^{\prime} \\
& -\nabla \nabla u\left(\xi^{\prime}, \xi^{\prime}\right),
\end{aligned}
$$

where, in coordinates,

$$
\nabla \nabla u\left(\xi^{\prime}, \xi^{\prime}\right)=u_{, j k}^{i} \xi^{\prime j} \xi^{\prime k}
$$

\section{A. Generalized Taylor or frozen turbulence hypothesis}

To derive the LANS- $\alpha$ equations, Marsden and Shkoller $^{31}$ used a generalization of the classical frozen turbulence hypothesis of Taylor. ${ }^{37}$ According to Taylor hypoth- 
esis the streamwise scalar component of the fluctuation is considered frozen over the time scale of the temporal derivative, giving

$$
\frac{\partial}{\partial t}=-U \frac{\partial}{\partial x}
$$

where $U$ is the local mean velocity along the streamwise direction, which is denoted by $x$.

The generalized Taylor hypothesis of Marsden and Shkoller ${ }^{31}$ consists of assumptions associated with behavior of the Lagrangian fluctuations at different orders of $\alpha$. Here we present their closure to $O\left(\epsilon^{2}\right)$, achieved by making the following assumptions:

$$
\begin{aligned}
& \partial_{t} \xi^{\prime}+(u \cdot \nabla) \xi^{\prime}-\left(\xi^{\prime} \cdot \nabla\right) u=0, \\
& \frac{D}{D t}\left\langle\xi^{\prime \prime}\right\rangle \perp u,
\end{aligned}
$$

where the orthogonality is taken in $L^{2}$ and, as usual,

$$
\frac{D}{D t} v=\partial_{t} v+(u \cdot \nabla) v
$$

Equation (6a) states that the Lagrangian fluctuation $\xi^{\prime}$ is Lie advected or frozen into the mean flow as a divergence-free vector field, i.e., $\partial_{t} \xi^{\prime}+£_{u} \xi^{\prime}=0$. Note that this equation automatically preserves the divergence free nature of $\xi^{\prime}$.

Substituting the relations (5a) and (5b) into the expansion (4) and using the generalized Taylor hypothesis (6a) and (6b), we find that

$$
u^{\epsilon}=u-\frac{1}{2} \epsilon^{2} \nabla \nabla u\left(\xi^{\prime}, \xi^{\prime}\right)+O\left(\epsilon^{3}\right) .
$$

Substitution of Eq. (7) into the averaged action function (3) gives

$$
\bar{S}=\frac{1}{2} \int_{0}^{T} \int_{\Omega}\left[|u|^{2}+\alpha^{2} \nabla \nabla u: F, u+O\left(\alpha^{3}\right)\right] d x d t,
$$

where the Lagrangian covariance tensor $F$ is defined by

$$
F=\left\langle\xi^{\prime} \otimes \xi^{\prime}\right\rangle \text {. }
$$

\section{B. The isotropic Lagrangian averaged Navier-Stokes (LANS- $\alpha$ ) equations}

In this paper we focus on the isotropic form of the LANS- $\alpha$ equations which is intended for modeling fluid flow away from boundaries. We start by assuming that the covariance tensor is isotropic

$$
F=c \mathrm{Id},
$$

a constant multiple of the identity. By integration-by-parts and truncation of the averaged action function to $O\left(\alpha^{2}\right)$, we find that

$$
\bar{S}^{\alpha}=\frac{1}{2} \int_{0}^{T} \int_{\Omega}\left[|u|^{2}+2 \alpha^{2}|D|^{2}\right] d x d t
$$

where

$$
D=\frac{1}{2}\left[\nabla u+(\nabla u)^{T}\right] .
$$

The superscript $\alpha$ denotes that the action $\bar{S}^{\alpha}$ depends on the parameter $\alpha$. Notice that the velocities $u$ obtained using this expression will also depend on parameter $\alpha$, however, we will not explicitly indicate this dependence in the following equations. We refer the reader to Marsden and Shkoller ${ }^{31}$ for the anisotropic case.

Applying the calculus of variations machinery to Eq. (8) yields the isotropic LANS- $\alpha$ equation

$$
\begin{aligned}
& \partial_{t} u+(u \cdot \nabla) u+\mathcal{U}^{\alpha, \text { iso }}(u)=-\left(1-\alpha^{2} \Delta\right)^{-1} \operatorname{grad} p-\nu A u, \\
& \operatorname{div} u=0, \quad u(0, x)=u_{0}(x), \\
& u=0 \text { on } \partial \Omega,
\end{aligned}
$$

where

$$
\begin{aligned}
\mathcal{U}^{\alpha, \text { iso }}(u)= & \alpha^{2}\left(1-\alpha^{2} \Delta\right)^{-1} \operatorname{Div}\left[\nabla u \cdot \nabla u^{T}+\nabla u \cdot \nabla u\right. \\
& \left.-\nabla u^{T} \cdot \nabla u\right] .
\end{aligned}
$$

Marsden and Shkoller ${ }^{21}$ showed that while this form of LANS- $\alpha$ equations is equivalent to that presented by Foias et $a l^{32,38,39}$ it reveals the additional boundary condition that must be satisfied when wall bounded flows are considered. The additional boundary condition is

$$
A u=0 \text { on } \partial \Omega .
$$

While this condition is naturally imposed upon the solution of the LANS- $\alpha$ equations on bounded domains, we believe that the anisotropic theory should be used to model turbulence in domains with boundaries. Here, $A$ is the Stokes operator $A=-P \Delta$ where $P$ is the Leray (or the HelmholtzHodge) projector. The most obvious scenario in which one might assume the covariance is isotropic is in decaying or forced turbulence inside a periodic box. In this case, the inverse Helmholtz operator commutes with the divergence and gradient operators, and the isotropic LANS- $\alpha$ equations are given by

$$
\begin{aligned}
& \partial_{t} u+(u \cdot \nabla) u=-\operatorname{grad} \tilde{p}+\nu \Delta u+\operatorname{Div} \tau^{\alpha}(u), \\
& \operatorname{div} u=0,
\end{aligned}
$$

where $u$ is the macroscopic velocity, $\nu$ is the kinematic viscosity, and $\tau^{\alpha}(u)$ can be identified as the subgrid stress tensor, defined by

$$
\begin{aligned}
\tau^{\alpha}(u)= & -\alpha^{2}\left(1-\alpha^{2} \Delta\right)^{-1}\left[\nabla u \cdot \nabla u^{T}-\nabla u^{T} \cdot \nabla u\right. \\
& \left.+\nabla u \cdot \nabla u+\nabla u^{T} \cdot \nabla u^{T}\right]
\end{aligned}
$$

or in the Cartesian coordinates

$$
\begin{aligned}
\tau_{i j}^{\alpha}= & -\alpha^{2}\left(1-\alpha^{2} \Delta\right)^{-1}\left[\frac{\partial u_{i}}{\partial x_{k}} \frac{\partial u_{j}}{\partial x_{k}}-\frac{\partial u_{k}}{\partial x_{i}} \frac{\partial u_{k}}{\partial x_{j}}+\frac{\partial u_{i}}{\partial x_{k}} \frac{\partial u_{k}}{\partial x_{j}}\right. \\
& \left.+\frac{\partial u_{k}}{\partial x_{i}} \frac{\partial u_{j}}{\partial x_{k}}\right] .
\end{aligned}
$$

In this equation, $\alpha$ is a scale of the rapid fluctuations in the flow map, below which wave activity is filtered by a nonlinear redistribution of energy. Notice that the third term on the right hand side is equivalent to the tensor-diffusivity model 
developed by Leonard. ${ }^{7}$ Also, notice that the divergence of the last term on the right hand side can be expressed as a gradient of a scalar

$$
\operatorname{Div}\left(\nabla u^{T} \cdot \nabla u^{T}\right)=\operatorname{grad}\left(\nabla u^{T}: \nabla u^{T}\right) .
$$

Therefore, this term can be absorbed into the modified pressure function $\tilde{p}$. On the periodic box, the pressure function is determined (up to a constant) from the incompressibility condition as a solution of the Poisson problem obtained by taking the divergence of the momentum equation.

We point out that LANS- $\alpha$ equations can be rewritten so that the stress terms take a form similar to the constitutive relation for second grade fluids $33,38,40,41$

$$
\frac{\partial u}{\partial t}+(u \cdot \nabla) u=\operatorname{Div} \sigma(u)
$$

where the stress, $\sigma$, includes both viscous and subgrid stresses

$$
\sigma(u)=-p I+2 \nu\left(1-\alpha^{2} \Delta\right) D+2 \alpha^{2} \dot{D} .
$$

Here, $\dot{D}$ is co-rotational Jaumann derivative

$$
\dot{D}=\partial D / \partial t+(u \cdot \nabla) D+D R-R D,
$$

where $R$ is a rate of rotation tensor defined as

$$
R=\frac{1}{2}\left[\nabla u-(\nabla u)^{T}\right] .
$$

The key difference between the constitutive relation for second grade fluids and the subgrid stress given by Eq. (18) stems from the viscous stress term, that is, the second term on the right hand side of Eq. (18), which in the case of the LANS- $\alpha$ equations includes an additional Helmholtz operator $\left(1-\alpha^{2} \Delta\right)$. Rivlin ${ }^{13}$ was the first to suggest that the constitutive relations for non-Newtonian fluids could be used to model turbulent stresses. Rivlin showed that unlike linear models, in which turbulent stress is linearly related to the rate of deformation tensor, nonlinear relations are able to represent normal stress effects observed in sheared turbulent flows. Following Rivlin's suggestion, Lumley ${ }^{42}$ and later Pope $^{43}$ explored and developed models for Reynolds stresses in the context of the RANS equations. This line of research was continued and expanded by Speziale. ${ }^{44,45}$ In addition to the phenomenological approach, nonlinear models for Reynolds stresses were also developed using statistical turbulence theories by Yoshizava ${ }^{46}$ who employed a direct interaction approximation approach and Rubenstein and Burton ${ }^{47}$ who used renormalization group theory. As we have seen earlier the tensor-diffusivity model developed by Leonard ${ }^{7}$ can also be considered as a nonlinear model. This model was combined with the Smagorinsky model and a priori tested by Clark et al. ${ }^{9}$ Recently the nonlinear model of Leonard was used in combination with the dynamic Smagorinsky model by Leonard and Wincklemans ${ }^{48}$ and Wincklemans et al. $^{49}$ in the LES of incompressible wall bounded flows. Lund and Novikov $^{50}$ carried out an extensive a priori study of a wide range of nonlinear constitutive relations as subgrid models. At the same time, Wong ${ }^{51}$ proposed a way to combine a nonlinear subgrid model together with the dynamic modeling procedure. Kosovic ${ }^{52}$ developed a phenomenological nonlin- ear model and used it in simulations of atmospheric boundary layers. ${ }^{53}$ This nonlinear model can reproduce normal stress effects and represent the backscatter of energy without allowing for negative eddy viscosity.

In addition to the relation between constitutive relations for second grade fluids and LANS- $\alpha$ equations, Oliver and Shkoller $^{33}$ also demonstrated a connection between the LANS- $\alpha$ equations and vortex methods. While phenomenological nonlinear subgrid stress models and the subgrid term in LANS- $\alpha$ equations share certain properties, the subgrid term in LANS- $\alpha$ arises as a consequence of Lagrangian averaging, and is not based on a phenomenological modeling assumptions. Due to the existence of a variety of subgrid models, mathematical properties of various ad hoc filtered Navier-Stokes equations which are often used in LES have not been studied in detail. Contrary to that, mathematical properties of LANS- $\alpha$ equations have been studied extensively and the proofs of global well-posedness and regularity of solutions even in the case of wall bounded flows have been presented by Foias, Holm, and Titi ${ }^{32}$ and Marsden and Shkoller. ${ }^{21}$ One of the main problems of subgrid modeling for LES is the problem of no-slip boundary conditions in simulations of high Reynolds number wall bounded flows. In order to avoid resolving the viscous sublayer, which would be equivalent to carrying out DNS, approximate boundary conditions employing wall functions and assuming a logarithmic velocity profile are usually imposed near the wall. While this approach yields satisfactory results when simple flow domain geometries are considered it cannot be easily generalized to transitional flows or complex flow geometries regularly encountered in engineering applications. In the context of the isotropic LANS- $\alpha$ equations, such an initialboundary value problem is well posed if the additional boundary condition (12) is introduced. For the anisotropic LANS- $\alpha$ equations, the degeneracy of the covariance matrix plays the role of the additional boundary condition. ${ }^{54}$ In future work, we shall determine if the LANS- $\alpha$ equations are a viable wall-bounded turbulence model. As the first step toward this work, in this study, we shall focus on isotropic turbulence simulations in a periodic box.

\section{NUMERICAL METHOD}

Our computational domain is a periodic cubic box of side $2 \pi$. In a numerical simulation of decaying turbulence, the size of the computational domain imposes an upper bound on the growth of the large scales in the flow. This is consistent with the observation in most experiments that the largest scales of motion are of the same order as the size of the experimental apparatus. Given the number of grid points and the size of the computational domain, the smallest resolved length scale or equivalently the largest wave number, $k_{\text {max }}$, is prescribed. In a three-dimensional turbulent flow the kinetic energy cascades in time to smaller, more dissipative scales. The scale at which viscous dissipation becomes dominant, and which represents the smallest scales of turbulence, is characterized by the Kolmogorov length scale $\eta$ (whose definition is recalled below). In a fully resolved DNS, the condition $k_{\max } \eta \gtrsim 1$ is necessary for the small scales to be 
adequately represented. Consequently, $k_{\max }$ limits the highest achievable Reynolds number in a direct numerical simulation for a given computational box.

The full range of scales in a turbulent flow for even a modest Reynolds number spans many orders of magnitude, and it is not generally feasible to capture them all in a numerical simulation. On the other hand, in turbulence modeling, empirical or theoretical models are used to account for the net effect of small scales on large energy-containing scales. In the next sections, the numerical simulations of forced and decaying homogeneous turbulence based on the full DNS, LES modeling, and the LANS- $\alpha$ modeling are presented.

Direct numerical simulations (DNS) of isotropic flows using pseudospectral methods was pioneered by the work of Orszag and Patterson ${ }^{55}$ and Rogallo. ${ }^{56}$ The accuracy in the calculation of the spatial derivatives that appear in the Navier-Stokes equations is improved substantially by using the pseudospectral technique as compared with the finite difference method. The core of the numerical method used in this study is based on a standard parallel pseudospectral scheme with periodic boundary conditions similar to the one described in Rogallo. ${ }^{56}$ The spatial derivatives are calculated in the Fourier domain, while the nonlinear convective terms are computed in the physical space. The flow fields are advanced in time in physical space using a fourth order Runge-Kutta scheme. The time step was chosen appropriately to ensure numerical stability. To eliminate the aliasing errors in this procedure the two thirds rule is used, so that the upper one third of wave modes is discarded at each stage of the fourth order Runge-Kutta scheme.

In addition to LANS- $\alpha$ computations, we also performed simulations using a dynamic SGS model (see, e.g., Germano et al. ${ }^{12}$ ). Germano et al. ${ }^{12}$ suggested a dynamic procedure in which the model coefficient of an arbitrary functional relationship, selected to represent the subgrid scale stress tensor, can be evaluated as part of the simulation. This procedure, applied to the Smagorinsky eddy-viscosity model, has proven quite versatile and is used here as a representative of a class of LES models. The filter aspect ratio in the dynamic LES model is a free parameter and the final result depends on the value of this parameter particularly in severe test cases such as the one considered here. The model coefficient in the dynamic LES is averaged over the whole computational domain. However, the LES computations were repeated for many filter aspect ratios (at least four), and the parameter that matched the best with the turbulence decay of the DNS data or the DNS energy spectra was used in the computations of the next sections.

There are three main characteristic length scales in an isotropic turbulent flow: the integral scale $l$ characterizing the energy containing scales is defined as

$$
l=\frac{3 \pi}{4} \frac{\int_{0}^{k_{\max }} \frac{E(k)}{k} d k}{\int_{0}^{k_{\max }} E(k) d k},
$$

where $E(k)$ is the energy spectrum function at the scalar wave number $k \equiv(\mathbf{k} \cdot \mathbf{k})^{1 / 2}$; the Kolmogorov microscale $\eta$ representative of dissipative scales is

$$
\eta=\left(\frac{\nu^{3}}{\epsilon}\right)^{1 / 4}
$$

where $\epsilon$ is the volume averaged energy-dissipation rate; and the Taylor microscale $\lambda$ characterizing the mixed energydissipation scales is defined as

$$
\lambda=\sqrt{\frac{u_{\mathrm{rms}}^{2}}{\left\langle\left(\partial u_{1} / \partial x_{1}\right)^{2}\right\rangle}},
$$

where $u_{\mathrm{rms}}$ is the root mean square value of each component of velocity defined as

$$
u_{\mathrm{rms}}^{2}=\frac{2}{3} \int_{0}^{k_{\max }} E(k) d k
$$

The time scale of the energy containing eddies is the largeeddy-turnover time $T$ defined as $T \equiv l / u_{\mathrm{rms}} .{ }^{57}$ The Taylor Reynolds number (defined based on the Taylor microscale) is

$$
\operatorname{Re}_{\lambda} \equiv \frac{u_{\mathrm{rms}} \lambda}{\nu}
$$

We expect that simulations which share the same value of both $\operatorname{Re}_{\lambda}$ and the nondimensional Kolmogorov length scale $k_{0} \eta$ should be identical in a statistical sense.

In the next sections, we present results of our numerical simulations of the LANS- $\alpha$ equations. We first demonstrate the characteristics of the nonlinear redistribution of energy in the LAE- $\alpha$ equations, and then present results of our viscous computations.

\section{THE LAGRANGIAN-AVERAGED EULER EQUATIONS}

In Sec. II, we argued that the LAE- $\alpha$ equations redistribute the energy content among the small scales through a nonlinear redistribution mechanism. This is illustrated in Fig. 1 where various energy norms in the LAE- $\alpha$ simulations (i.e., no viscous dissipation) are contrasted against the energy norm in the Euler calculation. The initial condition is the same as the one described in Sec. IV A for the viscous computations based on the initial data from Comte-Bellot and Corrsin ${ }^{28,29}$ test case (CBC). As expected, the $H^{1}$-equivalent energy norm for the LAE- $\alpha$ equations given by

$$
H^{1}(u)=\frac{1}{2} \int\left[u \cdot u+2 \alpha^{2} D \cdot D\right] d^{3} x,
$$

is a conserved quantity. $D$ is defined in Eq. (9). In the case of periodic boundary conditions, this energy function may also be expressed as $H^{1}=\frac{1}{2} \int u \cdot\left(1-\alpha^{2} \Delta\right) u$. The value of $H^{1}$ depends on the initial condition, as well as the parameter $\alpha$. For given initial data and fixed $\alpha$, on the other hand, the $L_{2}$ energy function

$$
L_{2}(u)=\frac{1}{2} \int u \cdot u d^{3} x
$$




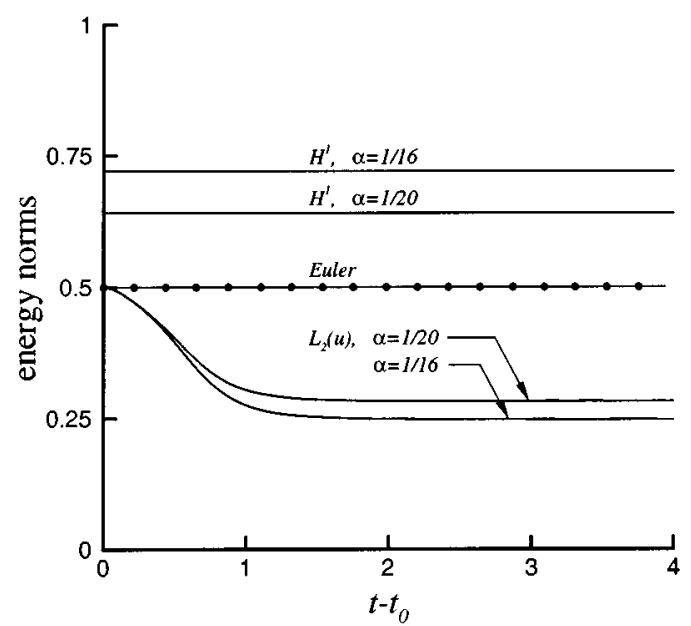

(a)

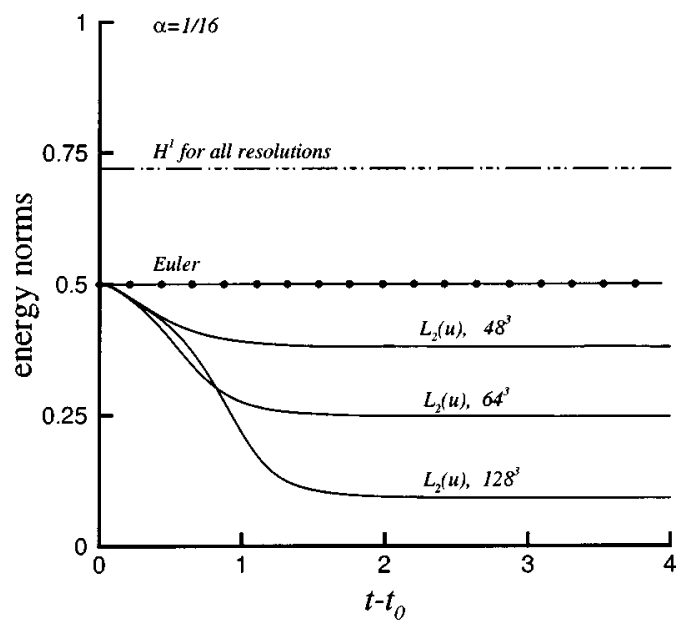

(b)

FIG. 1. Euler and the LAE- $\alpha$ simulations starting with CBC initial data at $t-t_{0}=1.8$. Euler (solid with circles) (a) $N_{\alpha}^{3}=64^{3}$ for $\alpha=1 / 16$, (b) $\alpha=1 / 16$; with $N_{\alpha}^{3}=48^{3}, 64^{3}$, and $128^{3}$. Note that the flat part of the $L_{2}(u)$ curves for the LAE- $\alpha$ cases is due to the finite resolution of the computational domain, and it is not a characteristic of the LAE- $\alpha$ equations.

drops significantly from its normalized initial value of 0.5 . It is precisely this quantity that we shall compare with the numerical simulation of Navier-Stokes equations. Again, the absolute drop in $L_{2}(u)$ depends on the initial velocity as well as the value of $\alpha$. Since $H^{1}(u)$ is constant along solutions of the LAE- $\alpha$ equations, any decay in $L_{2}(u)$ is followed by an increase in $\alpha^{2} L_{2}(\nabla u)$. After some initial transient all of the energy norms saturate. We remark that when $\alpha$ goes to zero, by definition the LANS- $\alpha$ computations approach DNS. In other words when $\alpha=0$, the $H^{1}$ energy norm (25) reduces to the usual $L_{2}$ norm for the kinetic energy.

In homogeneous flows the following equality holds

$$
2 D \cdot D=\omega \cdot \omega,
$$

where $\omega$ is a vorticity vector, $\omega=\nabla \times u$. It follows that in such flows the $H^{1}$ norm can be expressed as a sum of the $L_{2}$ norm and enstrophy (the $L_{2}$ norm of vorticity) multiplied by a factor $\alpha^{2}$.

$$
H^{1}(u)=L_{2}(u)+\alpha^{2} \mathcal{E}(u) .
$$

Here, enstrophy is defined as

$$
\mathcal{E}(u)=L_{2}(\omega)=\frac{1}{2} \int \omega \cdot \omega d^{3} x .
$$

Therefore a drop in kinetic energy ( $L_{2}$ norm) is accompanied by a proportional increase in enstrophy.

In a viscous computation the decay in $L_{2}(u)$ is augmented by the viscous decay in $L_{2}(u)$, as the viscous effects remove energy from small scales. Viscous computations are performed in the next sections to quantify the nature of the viscous decay.

In Fig. 1(b) the effect of grid resolution on the LAE- $\alpha$ simulations is shown. While the value of $H^{1}(u)$ is the same in both $48^{3}, 64^{3}$, and $128^{3}$ runs, the $L_{2}$ norm of $u$ and $\nabla u$ are significantly different after an initial transient. Therefore the decay in $L_{2}(u)$ depends on the size of the computational domain. One should note that the conservation of the $H^{1}$ norm is slightly sensitive to the accuracy of the implemented numerical scheme. The fourth order Runge-Kutta has proved to be adequate in our case. However, numerical experimentation has shown that lower-order time integration schemes might result in a slight decay in the $H^{1}$ norm. All of the reported computations in this study are performed using the same fourth order Runge-Kutta scheme.

\section{FORCED TURBULENCE}

Forced isotropic turbulence in a periodic box can be considered as one of the most basic numerically simulated turbulent flows. Forced isotropic turbulence is achieved by applying isotropic forcing to the low wave number modes so that the turbulent cascade develops as the statistical equilibrium is reached. Statistical equilibrium is signified by the balance between the input of kinetic energy through the forcing and its output through the viscous dissipation. Isotropic forcing cannot be produced in a laboratory and therefore forced isotropic turbulence is an idealized flow configuration that can be achieved only via a controlled numerical experiment; nevertheless, forced isotropic turbulence represents an important test case for studying basic properties of turbulence in a statistical equilibrium.

The numerical forcing of a turbulent flow is usually referred to the artificial addition of energy to the large scale (low wave number) velocity components in the numerical simulation. Forcing of the large scales of the flow is often used to generate a statistically stationary velocity field, in which the energy cascades to the small scales and is dissipated by viscous effects. In the statistically stationary state, the average rate of energy addition to the velocity field is equal to the average energy-dissipation rate. The Reynolds number attainable for a given size of simulation is substantially higher for forced turbulence than for the case of decaying turbulence.

It is generally believed that the Kolmogorov cascade theory ${ }^{58}$ provides an approximate description of homogeneous isotropic turbulence. The almost universal scaling of 
the dissipation range in Kolmogorov variables and the $k^{-5 / 3}$ energy spectrum are among the most successful predictions in turbulent flows. It is expected that any turbulent model must accurately predict this energy spectrum. Statistically stationary and homogeneous turbulence provides an ideal framework to study the capabilities of any turbulence model in predicting the Kolmogorov energy spectrum. To this end Chen et $a l .{ }^{26}$ numerically investigated the LANS- $\alpha$ equations in the case of forced homogeneous turbulence with a forcing technique similar to the forcing scheme $B$ implemented in this study. They concluded that the LANS- $\alpha$ simulations accurately predicts the Kolmogorov energy spectrum for scales larger than the parameter $\alpha$. However, one might attribute their results to the nature of their forcing technique; that is forcing based on the Kolmogorov scaling $k^{-5 / 3}$ in the forced region. In this section we repeat their numerical experiment, expand it and perform a new set of forced calculations with a different forcing technique that does not have the limitations of the forcing scheme implemented in Chen et al. ${ }^{26}$ The resolutions of all of our forced DNS computations are $170^{3}$ ( $256^{3}$ before dealiasing).

\section{A. Forcing schemes}

In using a low wave number forcing, we assume that the time averaged small scale quantities are not influenced by the details of the energy production mechanisms at large scales. This assumption is closer to the truth at high Reynolds numbers, where the energy containing scales are widely separated from the dissipation scales. The forcing parameters influence both the small scale quantities and the flow quantity variables such as the Reynolds number and the Kolmogorov length scale; however, a physically plausible forcing scheme should not influence the small scales independently of the flow quantity variables.

There are many methods for forcing homogeneous turbulent flows. Siggia and Patterson ${ }^{59}$ forced their simulations by applying a constant value for the amplitude of the Fourier coefficients $\hat{u}$ in the shell $1 \leqslant k \leqslant 2$. Eswaran and Pope ${ }^{60}$ implemented a different method. They used an UhlenbeckOrnstein stochastic diffusion process $w$ correlated over time with a chosen time scale to generate the random forcing of the form $\hat{f}_{i}(\mathbf{k}, t)=\left(\delta_{i j}-k_{i} k_{j} / k^{2}\right) w_{j}(\mathbf{k}, t) \times[\Theta(\mathbf{k})-\Theta(\mathbf{k}$ $\left.-\mathbf{k}_{\mathbf{F}}\right)$ ] where $\delta_{i j}$ is the Kronecker delta function and $\Theta$ is the Heaviside function. However, the velocity-force correlation contributes to the net forcing because there is a certain time scale over which the force is correlated. Ghosal et al. ${ }^{61}$ implemented a technique to guarantee that the production is balanced by the desired value of dissipation. They used a volume force of the form $\hat{\mathbf{f}}(\mathbf{k})=\epsilon \hat{u} /\left(N \hat{u}^{2}(\mathbf{k})\right)$ at the wave number $\mathbf{k}$ in the shell $k=k_{0}$ containing $N$ wave numbers where $\epsilon$ is the dissipation rate. Instead of forcing all the wave numbers in the shell $k=k_{0}$ Carati et al. ${ }^{62}$ forced only a randomly chosen wave numbers $N^{\prime}<N$ to eliminate the strong correlation of the forcing with the velocity field. Clearly, the external force injects the energy at the constant rate $\int \mathbf{f} \cdot \mathbf{u} d \mathbf{x} / V=\Sigma \mathbf{f}_{\mathbf{k}} \cdot u_{\mathbf{k}}=\boldsymbol{\epsilon}$ observed in Ghosal et al. ${ }^{61}$

Here we adopt two well-studied forcing methods that are different than the ones discussed above.

\section{B. Forcing scheme A}

The first method consists of applying forcing over a spherical shell with shell walls of unit width centered at wave number one, such that the total energy injection rate is constant in time. This forcing procedure was used by Misra and Pullin. ${ }^{63}$ The forcing amplitude is adjustable via the parameter $\delta$ while the phase of forcing is identical to that of the velocity components at the corresponding wave vectors. The Fourier coefficient of the forcing term is written as

$$
\hat{f}_{i}=\frac{\delta}{N} \frac{\hat{u}_{i}}{\sqrt{\hat{u}_{k} \hat{u}_{k}^{*}}}
$$

for all the modes in the specified shell. Here, $\hat{f}_{i}$ and $\hat{u}_{i}$ are Fourier transforms of the forcing vector $f_{i}$ and velocity, $u_{i}$, in the momentum conservation equation, $N$ is the number of wave modes that are forced. The above form of forcing ensures that the energy injection rate, $\sum \hat{f}_{i} \cdot \hat{u}_{i}$, is a constant which is equal to $\delta$. We chose $\delta=0.1$ for all of our runs.

\section{Forcing scheme B}

The second method corresponds to the forcing used by Chen and Shan $^{64}$ where wave modes in a spherical shell $|\mathbf{k}|=k_{0}$ of a certain width are forced in such a way that the forcing spectrum follows Kolmogorov's $-5 / 3$ scaling law

$$
\hat{f}_{i}=\frac{\delta}{N} \frac{\hat{u}_{i}}{\sqrt{\hat{u}_{k} \hat{u}_{k}^{*}}} k^{-5 / 3}
$$

this is done in order to obtain as long a range of near-inertial behavior as possible. This type of forcing ensures that the energy spectrum assumes inertial range scaling starting from the lowest wave modes and thus an extended inertial range is artificially created. Imposing inertial range scaling is particularly useful for studying inertial range transfer as well as scaling laws at higher wave numbers. We have chosen $k_{0}$ $=2$ and $\delta=0.03$ in all the runs.

\section{Results for forcing scheme A}

To present the results in a nondimensional form, we use the integral length scale $l$ and the root mean square of velocity $u_{\text {rms }}$. Throughout the forced simulations, these two quantities vary significantly; however, as equilibrium is approached, the integral length scale for the simulation with forcing scheme A approaches the value of approximately $l$ $\approx 0.2$, and the root mean square of velocity is $u_{\mathrm{rms}} \approx 0.25$. It follows, that the corresponding eddy-turnover time is $T$ $=l / u_{\mathrm{rms}}=0.8$. The computations are continued for more than 30 eddy-turnover times. The equilibrium Taylor Reynolds number in this simulation is approximately 80 .

The energy spectra at the nondimensional time $t=30.5$ is depicted in Fig. 2. It is interesting to note that for fixed value of $\alpha$ the lower resolution LANS- $\alpha$ cases $\left(64^{3}\right)$ are good representations of the higher resolution LANS- $\alpha$ cases $\left(170^{3}\right)$ for a large range of wave numbers. The low wavemodes of the DNS energy spectra are well captured by the LANS- $\alpha$ simulations with the expected trend; as $\alpha \rightarrow 0$ the LANS- $\alpha$ simulations approach the DNS spectra over the fully re- 


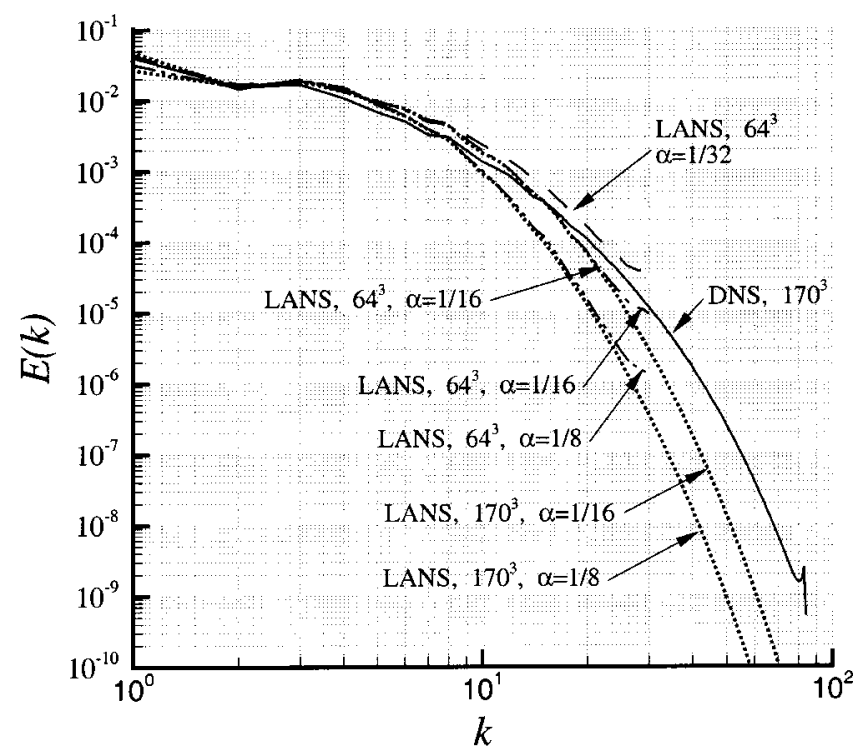

FIG. 2. The energy spectra at the nondimensional time $t=25 / 0.82=30.5$ for forcing scheme A.

solved wavemodes. The total kinetic energy $\left(L_{2}(u)\right)$ for the DNS and LANS- $\alpha$ computations are shown in Fig. 3. In these runs the flow is initialized from a fully developed turbulent flow at a higher Reynolds number. Consequently, the total kinetic energy drops as time evolves. The total kinetic energy passes through an initial transient state but eventually reaches a relative steady state. The plot of flatness and skewness of the DNS run (shown in Fig. 4) and the plot of the total kinetic energy indicate that after 10 eddy turnover times the flow is essentially in statistical equilibrium.

\section{E. Results for forcing scheme B}

The integral length scale for this run is approximately $l$ $\approx 0.32$, and the root mean square of velocity is $u_{\mathrm{rms}} \approx 1.4$. Consequently the eddy-turnover time can be calculated as $T=l / u_{\text {rms }}=0.23$. All of the computations for the forcing scheme B are continued for almost 25 eddy-turnover time

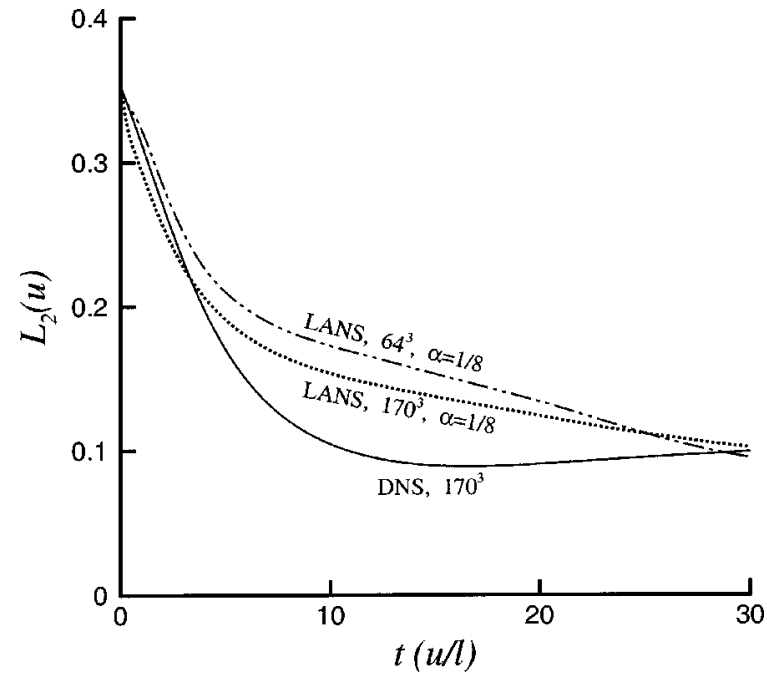

FIG. 3. Total kinetic energy for forcing scheme A.

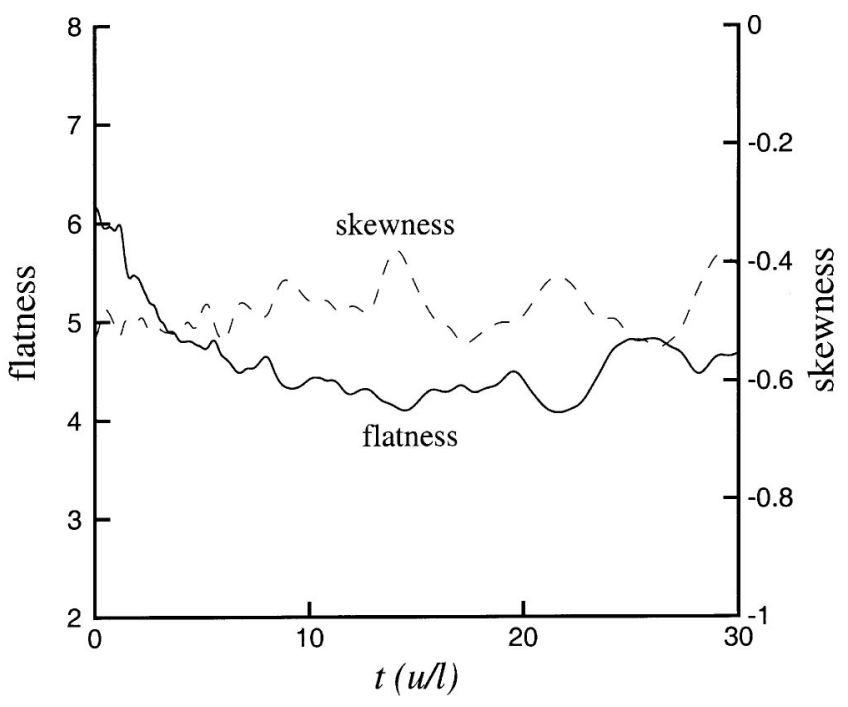

FIG. 4. Flatness and skewness for forcing scheme A in the DNS run.

units. The Taylor Reynolds number, $R_{\lambda}$, is approximately 115 for these computations. The energy spectra at the nondimensional time 14.1 are shown in Fig. 5. There is a well developed $k^{-5 / 3}$ region over one decade in wave numbers. The LANS- $\alpha$ calculations at the same full resolution follows the same behavior up to the spatial scale of order $\alpha$, where the inverse Helmholtz operator in the LANS- $\alpha$ computations sharply steepens the energy cascades to smaller scales. While it takes a relatively long time for the total kinetic energy to settle (see Fig. 6), the values of the skewness and the flatness reach an almost statistical equilibrium at around time $t \approx 5$, as shown in Fig. 7.

\section{F. Subgrid energy transfer and alignment of subgrid stress eigenvectors}

In order to evaluate the performance of any SGS model, it is (in principle) possible to compare the modeled SGS

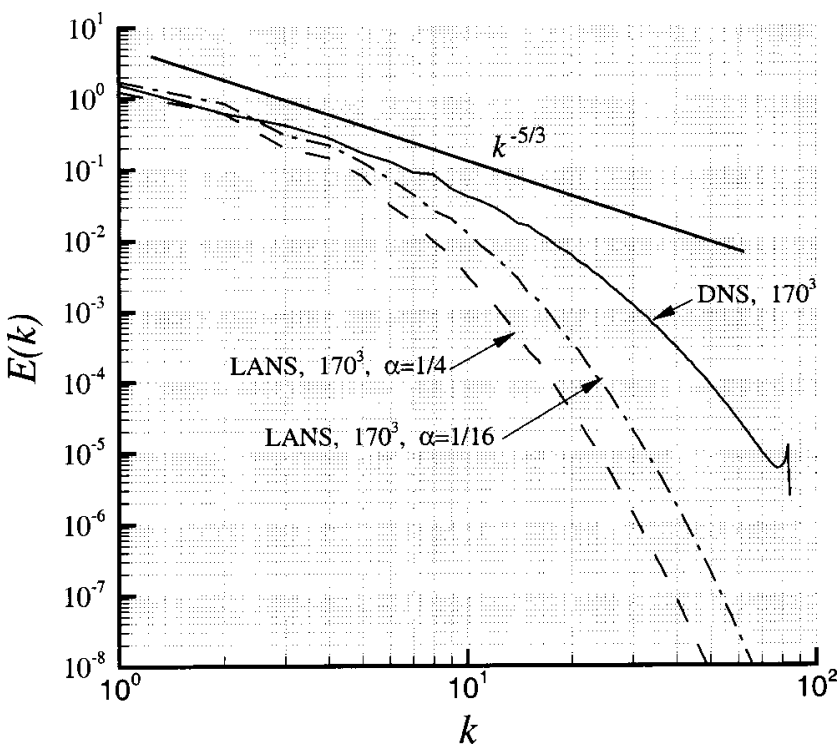

FIG. 5. The energy spectra at the nondimensional time $t=3.25 / 0.23=14.1$ for forcing scheme $\mathrm{B}$. The straight line represents a slope of $-5 / 3$. 


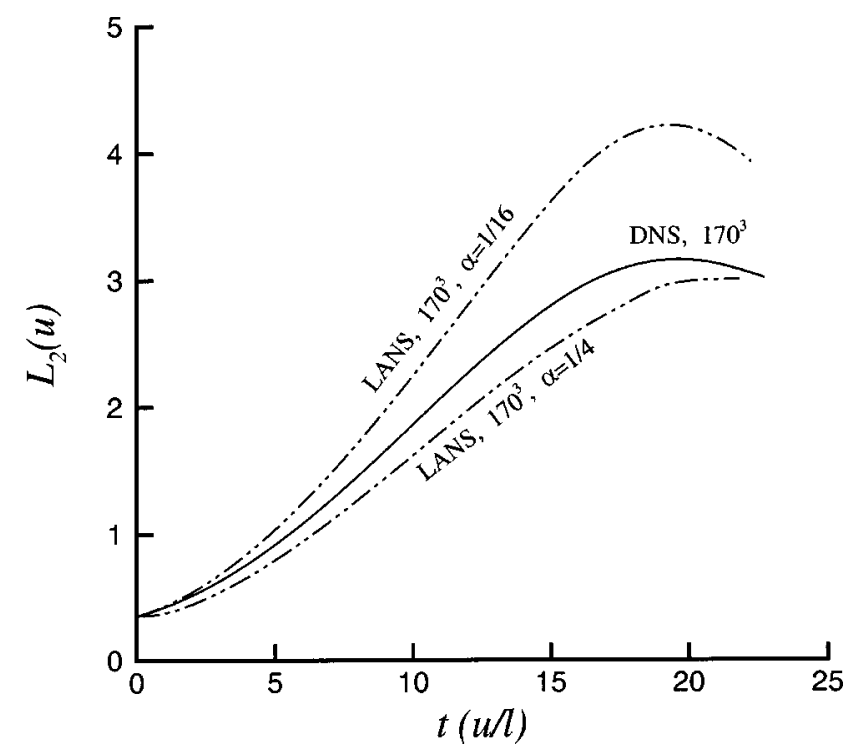

FIG. 6. Total kinetic energy for forcing scheme B.

stress $\tau^{\alpha}$ with the corresponding stress obtained by filtering DNS and computing correlation coefficients between SGS stress components. However, Piomelli et al. ${ }^{65}$ demonstrated that such an a priori technique does not account for the dynamic effects of the subgrid models and therefore cannot represent a definitive test of a subgrid model.

With the development of novel experimental techniques and the availability of high resolution DNS data, it is now possible to study basic structural properties of subgrid stresses with respect to the large scale characteristics of the flow field. A general feature of homogeneous isotropic turbulence is the observation of the tubelike fine scale eddies, ${ }^{66-68}$ which are considered as the smallest structure related to the intermittency of turbulence. The fine scale structures of turbulence have been one of the most important subjects in turbulence research. A comprehensive knowledge of the fine scale motions is essential in the development of a proper turbulent theory and any turbulent model. These co-

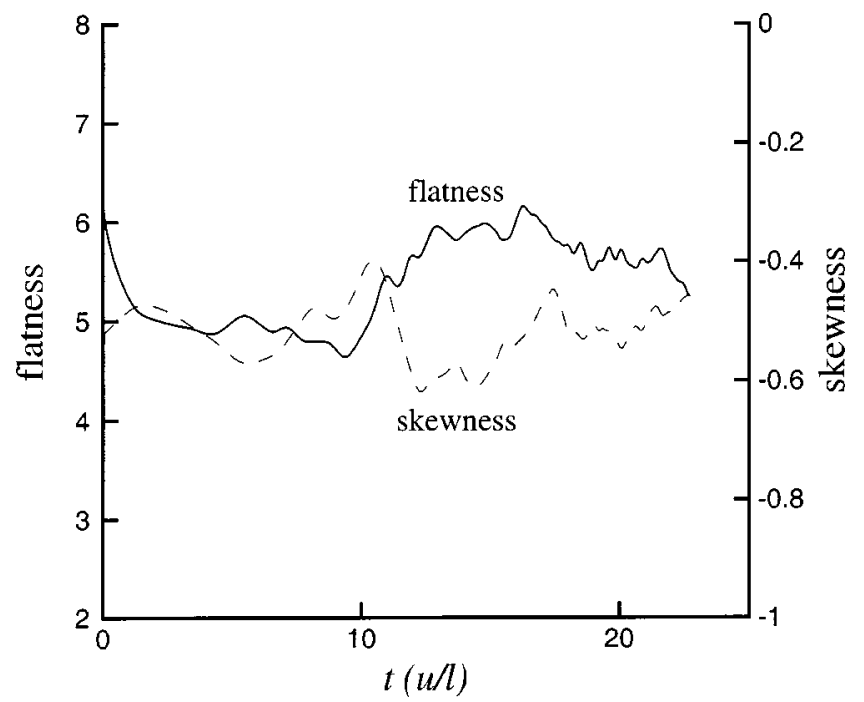

FIG. 7. Flatness and skewness for forcing scheme B in the DNS run. herent fine scale structures have been observed in other types of turbulent flows such as turbulent mixing layers and turbulent channel flows where they exhibited similar characteristics. $^{69,70}$

A three-dimensional measurement technique such as holographic particle velocimetry (HPIV) was used by Tao et $a .^{71-73}$ to study the alignment of the eigenvectors of actual and modeled components of the subgrid stresses as well as the alignments between eigenvectors of the rate of strain tensor and vorticity vector. They confirmed the preferred local alignment of the eigenvector of the rate of strain tensor corresponding to the intermediate eigenvalue with the vorticity vector, which was previously observed using pointwise DNS data. ${ }^{74,75}$ They also found a preferred relative angle between the most compressive eigendirection of the rate of strain tensor and the most extensive eigendirection of the SGS tensor.

Following the work of Tao et al., we will discuss the statistics of alignment between the eigenvectors of the SGS tensor $\tau^{\alpha}$, and both the eigenvectors of the rate of strain tensor, $D$, and the unit vorticity vector, $\boldsymbol{\omega}$, respectively defined by Eq. (9) and

$$
\boldsymbol{\omega}=\frac{\nabla \times u}{|\nabla \times u|} .
$$

The actual SGS tensor, $\tau$, can be computed by filtering the DNS data and using the definition

$$
\tau=(\overline{u \otimes u}-\bar{u} \otimes \bar{u}) .
$$

Here, overline denotes filtered quantities. We filter the DNS data by applying a wave cut-off filter with cut-off wave number $k_{c}=21$, corresponding to the largest wave number resolved in LANS- $\alpha$ simulation with $48^{3}$ grid points.

We denote the eigenvectors of $D$ by $\left[\mathbf{e}_{1}, \mathbf{e}_{2}, \mathbf{e}_{3}\right]$, ordered according to the corresponding eigenvalues $\left(\lambda_{1}, \lambda_{2}, \lambda_{3}\right)$, with $\lambda_{3}<\lambda_{2}<\lambda_{1}$. The eigenvectors of $\tau^{\alpha}$ are $\left[\mathbf{t}_{1}, \mathbf{t}_{2}, \mathbf{t}_{3}\right]$ with eigenvalues $\left(\gamma_{1}, \gamma_{2}, \gamma_{3}\right)$, such that $\gamma_{3}<\gamma_{2}<\gamma_{1}$. Thus, for example, we refer to $\widetilde{\mathbf{e}}_{1}$ as the most extensional eigendirection of $D$, and to $\mathbf{t}_{3}$ as the most compressive eigendirection of $\tau^{\alpha}$. Also, $\widetilde{\mathbf{e}}_{2}, \mathbf{t}_{2}$ are intermediate eigendirections with corresponding intermediate eigenvalues $\lambda_{2}, \gamma_{2}$, respectively.

We first use $170^{3}$ DNS data obtained using forcing scheme A and the data from a $48^{3}$ LANS- $\alpha$ simulation to compute the distribution of the energy transfer, $\epsilon_{s g s}=\tau: D$, due to the contribution of the subgrid term. The distributions are presented in Fig. 8. Note that $\epsilon_{s g s}<0$ corresponds to backscatter. In Fig. 9, the same energy transfer is computed for the cases with the forcing scheme B. Slightly skewed distributions presented in these figures with a significant portion of negative energy transfer indicate not only that the LANS- $\alpha$ model is capable of predicting backscatter of energy, but also that it can predict the probability distribution of the level of backscatter accurately.

Using the same data sets we computed the distributions of the alignment (cosine of the angles) between the eigenvectors of the rate of strain tensor and vorticity vector as well as the SGS tensor. We first use forcing scheme A and the LANS- $\alpha$ simulations with $64^{3}$ grid points and $\alpha=1 / 8$. Fig- 


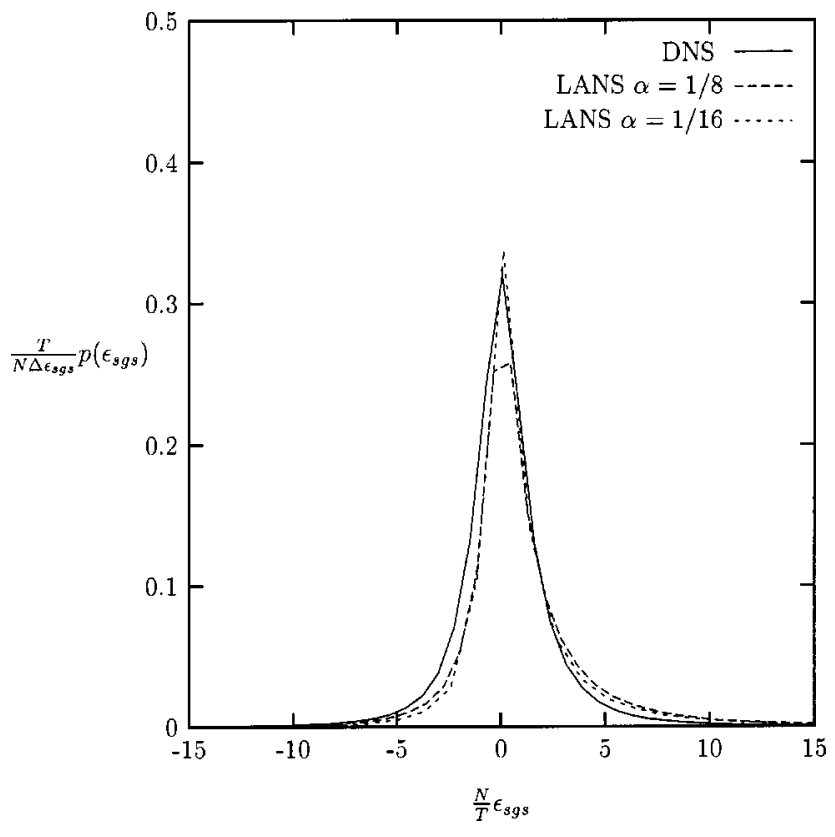

FIG. 8. Probability distribution of $\epsilon_{s g s}=\tau: D$; solid line-forced DNS $170^{3}$-forcing scheme $\mathrm{A}, \mathrm{Re}_{\lambda}=80$; dotted line-forced LANS- $\alpha 48^{3}$ with $\alpha=1 / 8$ and $\alpha=1 / 16$-forcing scheme A. The scalings are done based on the energy transfer $T$, sample size $N$, and the bin size $\Delta \epsilon_{s g s}$.

ures 10 and 12 indicate that in the LANS- $\alpha$ simulations, the preferential alignment between the vorticity vector and the eigendirection of the rate of strain tensor corresponding to the intermediate eigenvalue is not as pronounced as in DNS. As can be seen in Figs. 11 and 13 the eigenvectors of the modeled subgrid tensor $\tau^{\alpha}$ display qualitatively similar alignment as that computed from DNS data. In particular, the experimentally observed preferred angle between $\mathbf{e}_{3}$ and $\mathbf{t}_{1}$ is

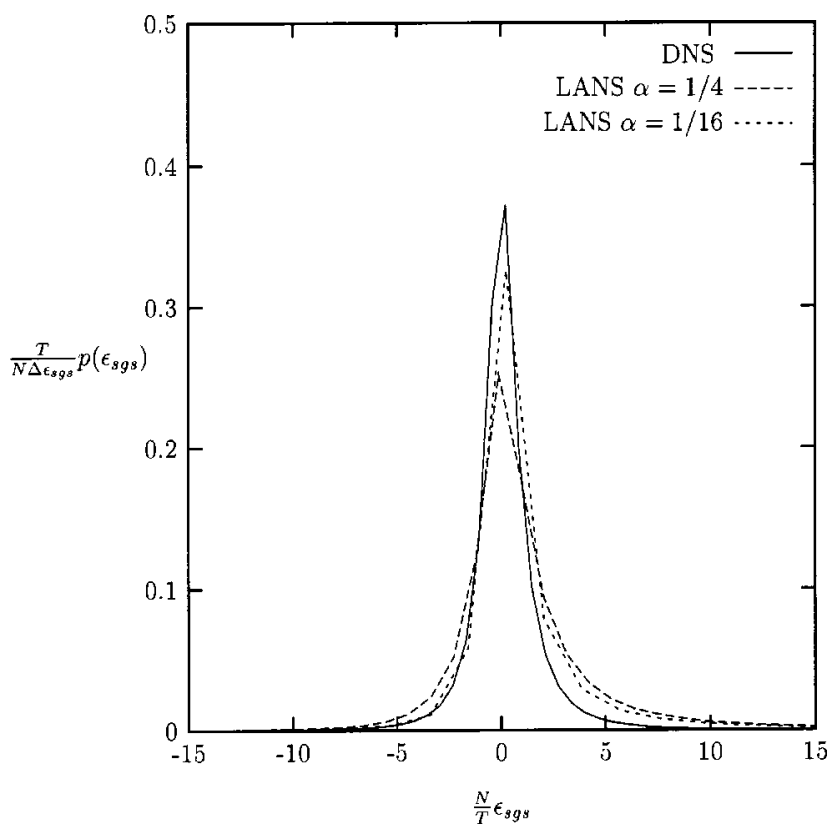

FIG. 9. Probability distribution of $\epsilon_{s g s}=\tau: D$; solid line-forced DNS $170^{3}$-forcing scheme $\mathrm{B}, \operatorname{Re}_{\lambda}=115$; dotted line-forced LANS- $\alpha 64^{3}$ with $\alpha=1 / 4$ and $\alpha=1 / 16$-forcing scheme B. The scalings are done based on the energy transfer $T$, sample size $N$, and the bin size $\Delta \epsilon_{s g s}$.

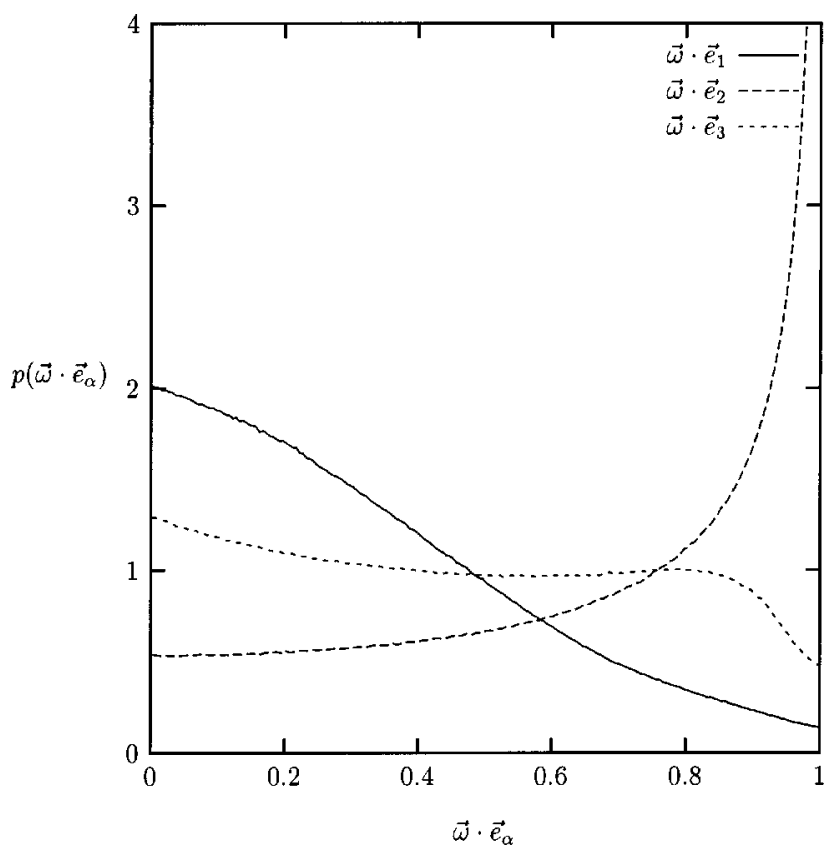

FIG. 10. Probability distribution of $\boldsymbol{\omega} \cdot \mathbf{e}_{\alpha}$; forced DNS $170^{3}$-forcing scheme $\mathrm{A} ; \mathrm{Re}_{\lambda}=80$.

captured by the LANS- $\alpha$ model. Similar observations can be made based on the simulations where the forcing scheme B was used. For these cases, probability distributions of the cosine of the angles between eigenvectors and the vorticity vector are obtained by filtering the DNS data using a wave cut-off filter with cut-off wave number $k_{c}=32$ and is given in Figs. 14 and 15. These results are compared to the LANS- $\alpha$ results corresponding to simulations with $64^{3}$ grid points and $\alpha=1 / 16$ which are presented in Figs. 16 and 17. We remark that commonly used linear, Smagorinsky-type,

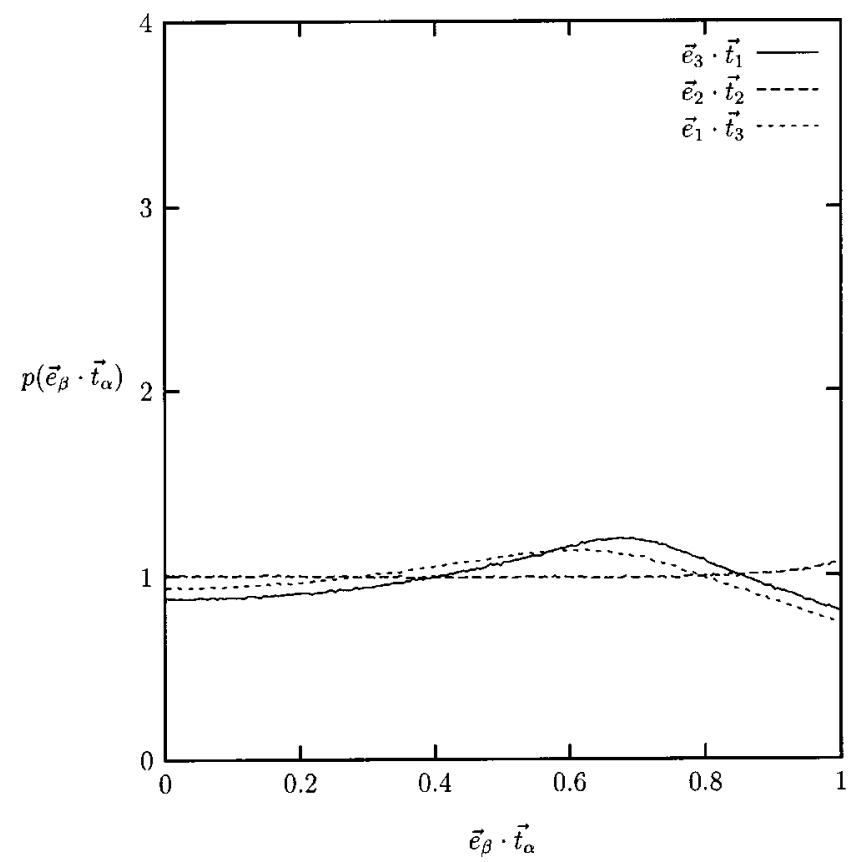

FIG. 11. Probability distribution of $\mathbf{e}_{1} \cdot \mathbf{t}_{3}, \mathbf{e}_{2} \cdot \mathbf{t}_{2}$, and $\mathbf{e}_{3} \cdot \mathbf{t}_{1}$; forced DNS $170^{3}$-forcing scheme $\mathrm{A} ; \mathrm{Re}_{\lambda}=80$. 


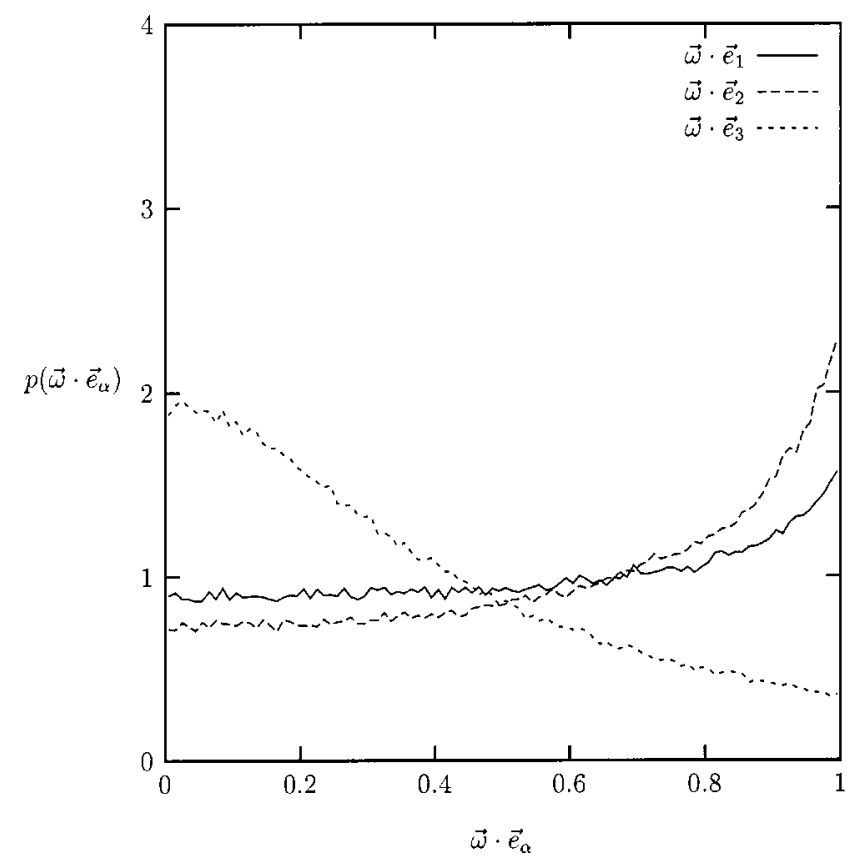

FIG. 12. Probability distribution of $\boldsymbol{\omega} \cdot \mathbf{e}_{\alpha}$; forced LANS- $\alpha 48^{3}$ with $\alpha$ $=1 / 8$-forcing scheme A.

SGS models implicitly assume that the eigenvectors of the SGS stress are locally aligned with the eigenvectors the eigenvectors of the rate of strain tensor, which is contrary to the experimental evidence.

\section{DECAYING TURBULENCE}

Decaying homogeneous turbulence is a more realistic idealization of a turbulent flow than the forced homogeneous case. Numerous theoretical, experimental, and computational studies followed the influential work of Taylor. ${ }^{76}$ The major-

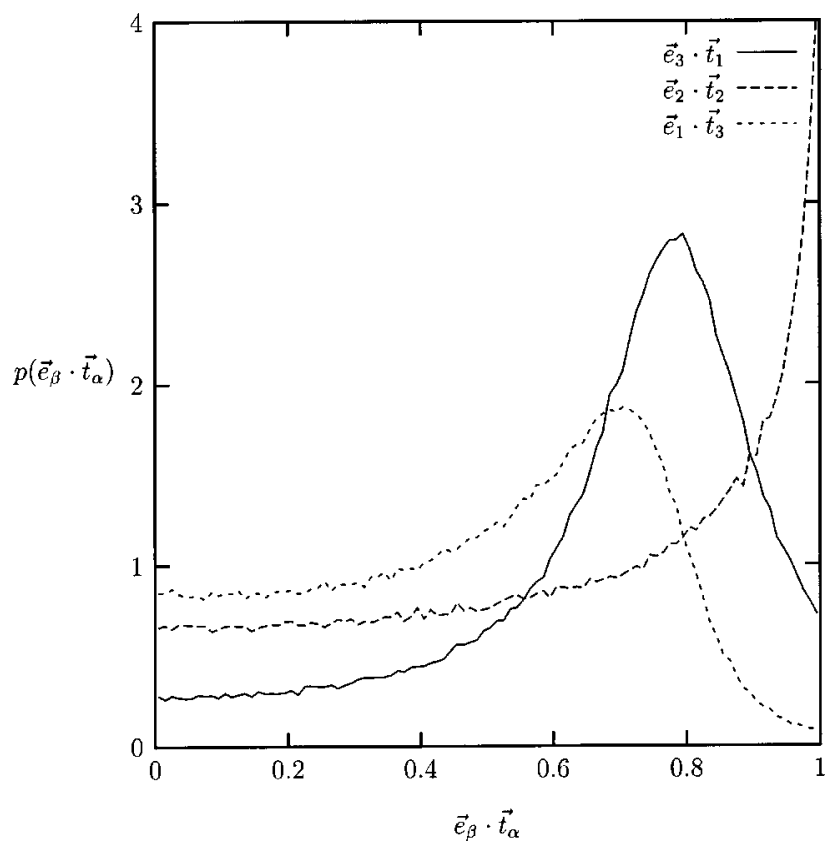

FIG. 13. Probability distribution of $\mathbf{e}_{1} \cdot \mathbf{t}_{3}, \mathbf{e}_{2} \cdot \mathbf{t}_{2}$, and $\mathbf{e}_{3} \cdot \mathbf{t}_{1}$; forced LANS- $\alpha 48^{3}$ with $\alpha=1 / 8$-forcing scheme A.

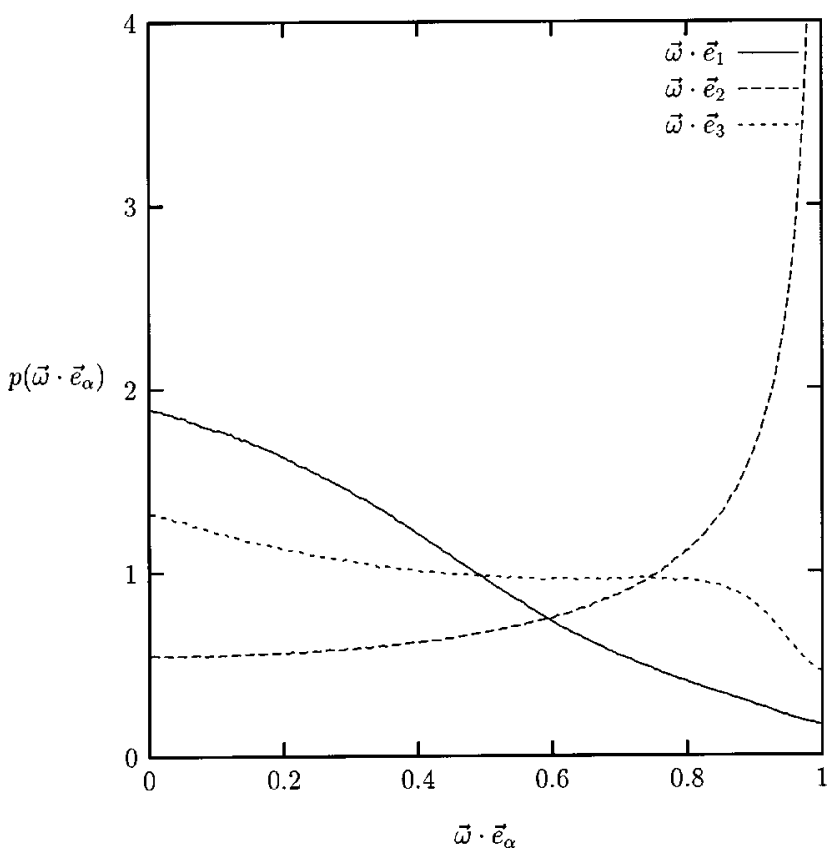

FIG. 14. Probability distribution of $\boldsymbol{\omega} \cdot \mathbf{e}_{\alpha}$; forced DNS $170^{3}$-forcing scheme $B ; \operatorname{Re}_{\lambda}=115$.

ity of the initiated experimental work was generated by a grid in wind tunnels, where the turbulence decays as the flow convects downstream. This grid-generated turbulence is regarded as homogeneous and isotropic some distance downstream by using special arrangements such as a secondary contraction of the test section. ${ }^{28}$ The comparison of experimental data with theory or computation assumes validity of the frozen turbulence hypothesis of Taylor. ${ }^{76}$ Other tech-

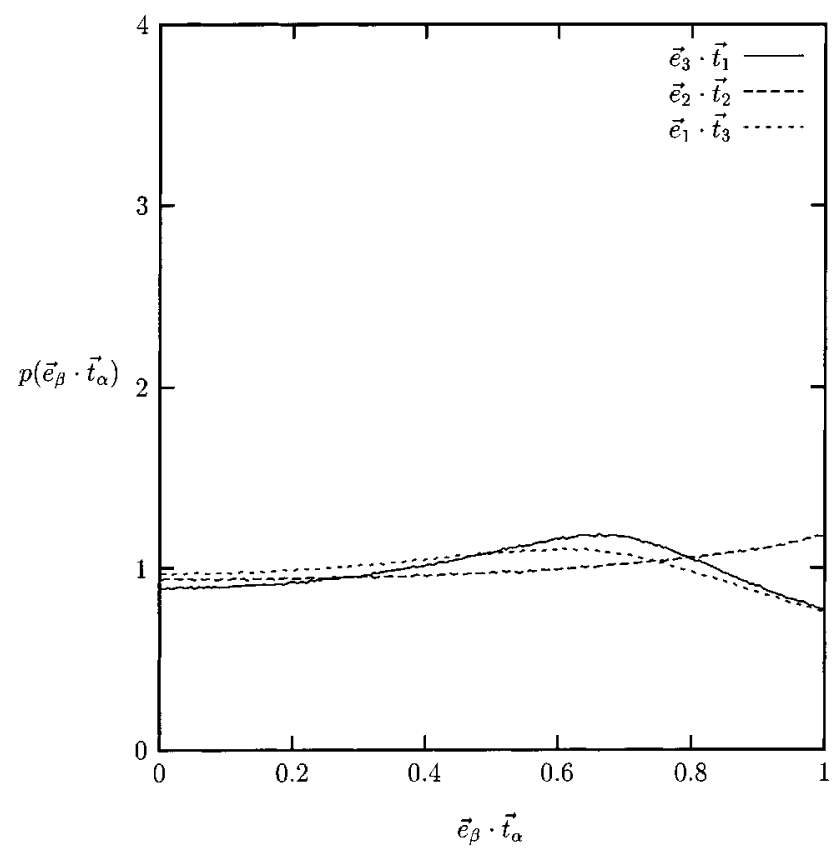

FIG. 15. Probability distribution of $\mathbf{e}_{1} \cdot \mathbf{t}_{3}, \mathbf{e}_{2} \cdot \mathbf{t}_{2}$, and $\mathbf{e}_{3} \cdot \mathbf{t}_{1}$; forced DNS $170^{3}$-forcing scheme $\mathrm{B} ; \mathrm{Re}_{\lambda}=115$. 


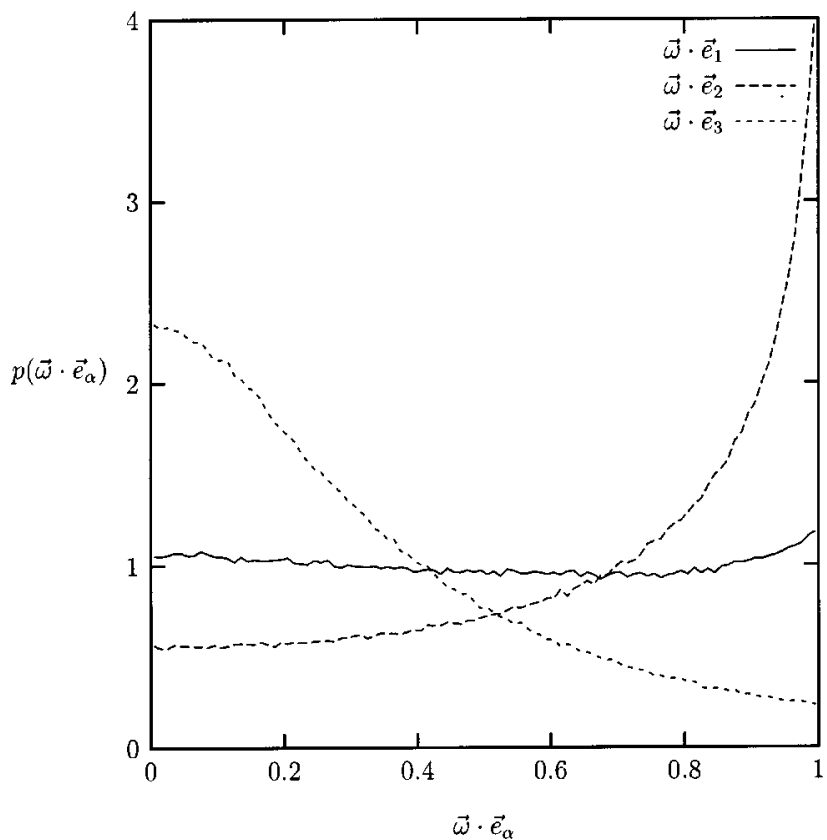

FIG. 16. Probability distribution of $\boldsymbol{\omega} \cdot \mathbf{e}_{\alpha}$; forced LANS- $\alpha 64^{3}$ with $\alpha$ $=1 / 16$ - forcing scheme B.

niques for generating turbulence without a mean flow are by towing a grid through a stationary sample of fluid $^{77}$ or by using an oscillating grid. $^{78}$

In this section we consider two different decaying turbulent flows. In the first case an initial condition from a realization of the experiments by Comte-Bellot and Corrsin ${ }^{28,29}$ is used. This initial condition has a broad energy spectrum with the peak of the energy spectrum at a relatively high wave number. In the second case we generate a sharply peaked initial energy spectrum with the peak of the energy

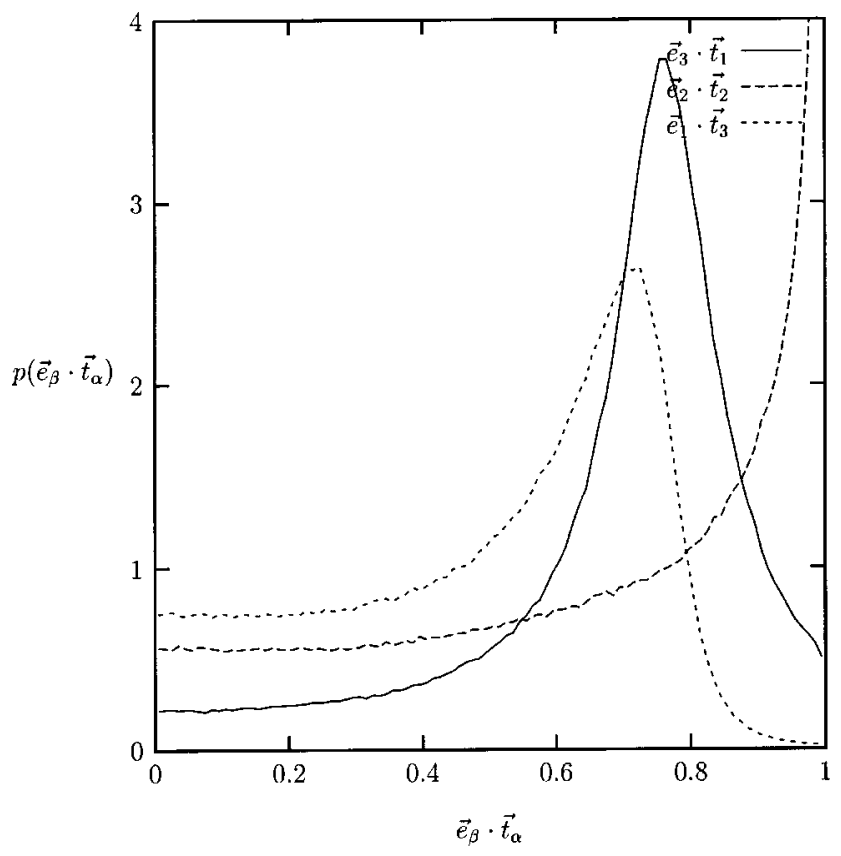

FIG. 17. Probability distribution of $\mathbf{e}_{1} \cdot \mathbf{t}_{3}, \mathbf{e}_{2} \cdot \mathbf{t}_{2}$, and $\mathbf{e}_{3} \cdot \mathbf{t}_{1}$; forced LANS- $\alpha 64^{3}$ with $\alpha=1 / 16$-forcing scheme B.

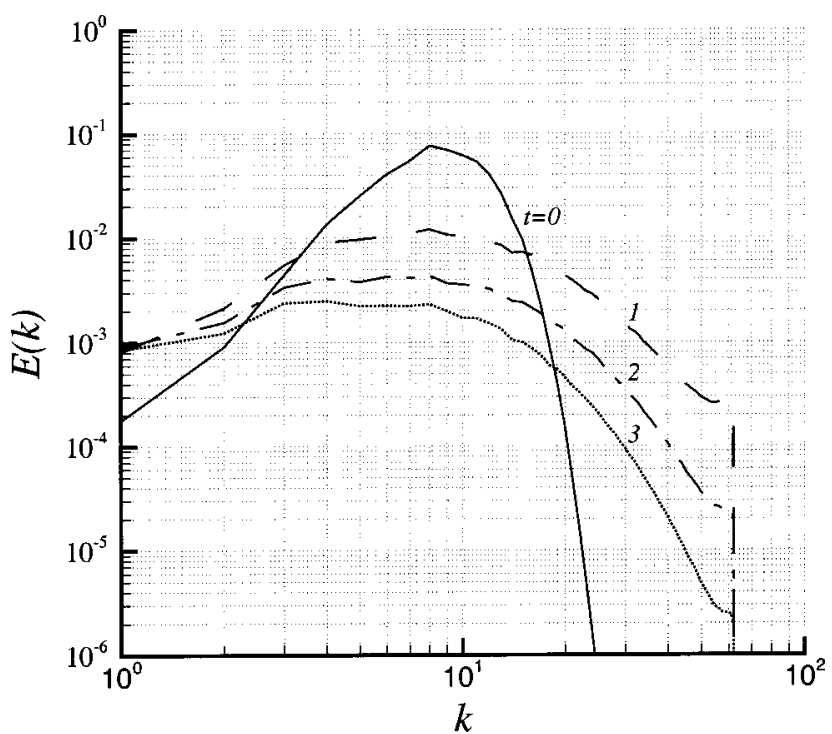

FIG. 18. Spectrum of the energy for the CBC initial data, $\operatorname{Re}_{\lambda}=72, N_{\mathrm{DNS}}^{3}$ $=128^{3}, t=0$ (solid); $t=1$ (dash); $t=2$ (dash dot); $t=3$ (dotted).

spectrum at a relatively low wave number. In this way the effects of the initial energy spectrum on the performance of the LANS- $\alpha$ calculations are investigated.

\section{A. Initial data from Comte-Bellot and Corrsin test case}

The most widely used published data on decaying grid turbulence are due to Comte-Bellot and Corrsin ${ }^{28,29}$ which we will hereafter refer to as CBC. Their data are well documented and has been used widely in the development of DNS, LES, and other turbulence models.

The initial field is taken from Wray. ${ }^{79}$ Wray provides a filtered velocity field in physical space, derived from $512^{3}$ data by a sharp truncation in Fourier space to $128^{3}$. All of the computations performed in this study were started with this data. The initial Taylor Reynolds number is $\operatorname{Re}_{\lambda}=72$. We increased the physical Reynolds number so that the evolution of this initial data is well resolved in a $128^{3}$ computation (before dealiasing it is $192^{3}$ ).

The evolution of the energy spectrum as predicted by DNS using $128^{3}$ points is illustrated in Fig. 18. The energy of fully developed isotropic turbulence decays in time while the scales of motion grow; the resulting $\operatorname{Re}_{\lambda}$ decreases with time. Consequently, a well resolved, fully developed field will remain well resolved as it decays. On the other hand, the integral scales grow in time and will eventually become comparable to the size of the computational box. Since the computational box contains only a small sample of the largest representable eddies, eventually the computation will suffer from a lack of sample in the energy-containing scales. The rather wide initial energy spectrum with a peak around the eighth Fourier mode provides a harsh test case for any turbulence model at such a resolution. The initial condition for LES and LANS- $\alpha$ simulations is obtained by spectrally filtering fully resolved velocity field to the resolution of these simulations. The best behavior of the LANS- $\alpha$ modeling is expected for applications in which the scale $\alpha$ is within, or at 


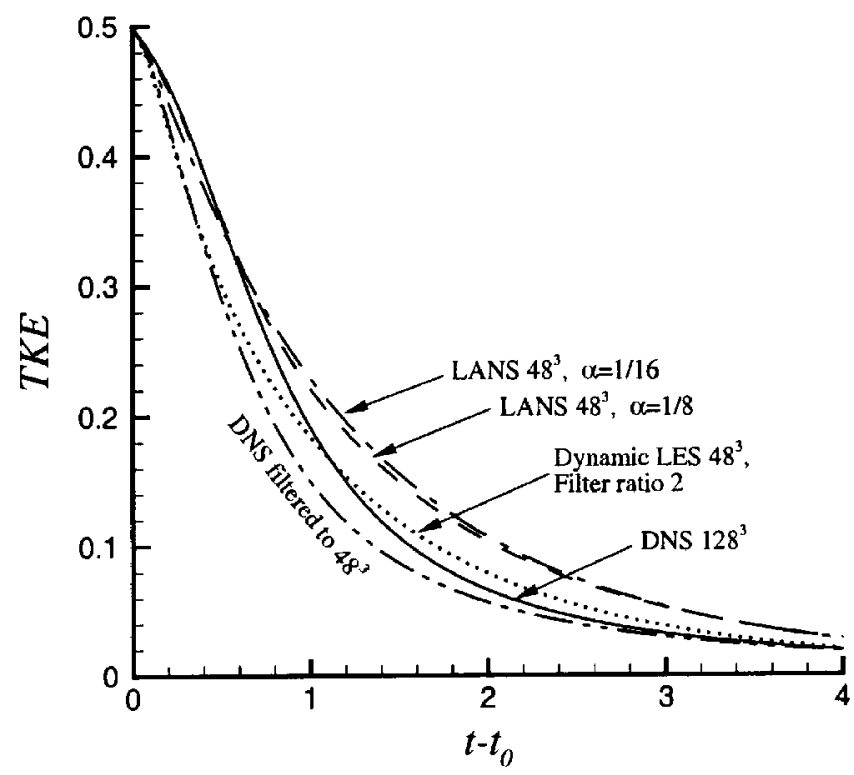

(a)

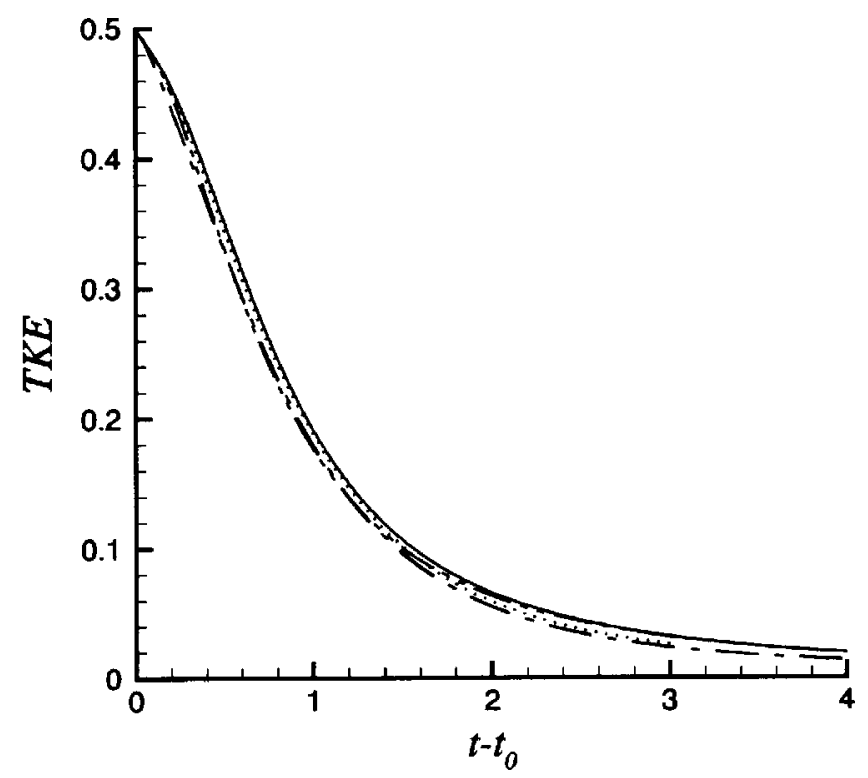

(b)

FIG. 19. Decay of kinetic energy with the CBC initial data, full DNS $128^{3}$ (solid); (a) $N_{\alpha}^{3}=48^{3}$, LANS- $\alpha$ with $\alpha=1 / 16$ (dash dot); LANS- $\alpha$ with $\alpha=1 / 8$ (dash); dynamic LES with a filter ratio 2 (dotted); DNS sharply filtered to $48^{3}$ (dash dot dot); (b) $N_{\alpha}^{3}=64^{3}$, LANS- $\alpha$ with $\alpha=1 / 16$ (dash); LANS- $\alpha$ with $\alpha=1 / 20$ (dash dot); dynamic LES with a filter ratio 4 (dotted); DNS sharply filtered to $64^{3}$ (dash dot dot). The filter aspect ratio in the dynamic LES is an extra free parameter. The dynamic LES computations were repeated for many filter aspect ratios (at least four), and only the results for the filter ratio with the best match with the DNS data are reported here (see also Fig. 20).

least close to, an inertial subrange. The $\mathrm{CBC}$ experiments at $\operatorname{Re}_{\lambda}=72$ barely satisfy this criterion and provide a severe test case of the LANS- $\alpha$ turbulence modeling capability.

The evolution of total kinetic energy (TKE) of the DNS data is contrasted against various dynamic LES and LANS- $\alpha$ simulations in Fig. 19, for two resolutions: $48^{3}$ and $64^{3}$. TKEs for DNS data, spectrally filtered to the resolution of the LES and LANS- $\alpha$ computations, are also presented. The filter aspect ratio in the dynamic LES model is a free parameter and the final result depends on the value of this parameter particularly in severe test cases such as the one considered here. In order to avoid introducing any further arbitrary parameters, the LES computations were repeated for many

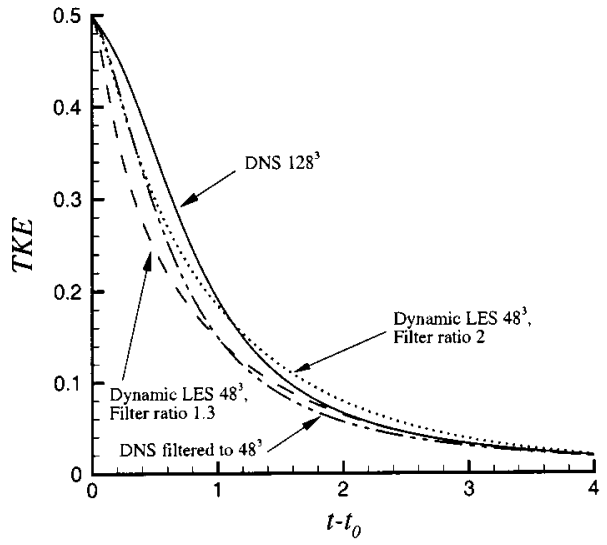

(a) filter aspect ratios, and the parameter that matched the best with the turbulence decay of the DNS data or the DNS energy spectra was used for comparison with the LANS- $\alpha$ equations. The best match between the DNS data and the dynamic LES results is achieved for a filter aspect ratio of 2 and 4 in the $48^{3}$ and $64^{3}$ calculations, respectively (see Fig. 20 ). In the $64^{3}$ computation both LES and LANS- $\alpha$ satisfactorily predict the decay rate. However, at the lower resolution of $48^{3}$ both models underestimate the decay rate, with the LANS- $\alpha$ model being more under-dissipative. It is clear that at such a low resolution the energy-containing part of the spectrum is barely resolved. This is demonstrated in Figs. 21-23 where the evolution of the energy spectrum of various

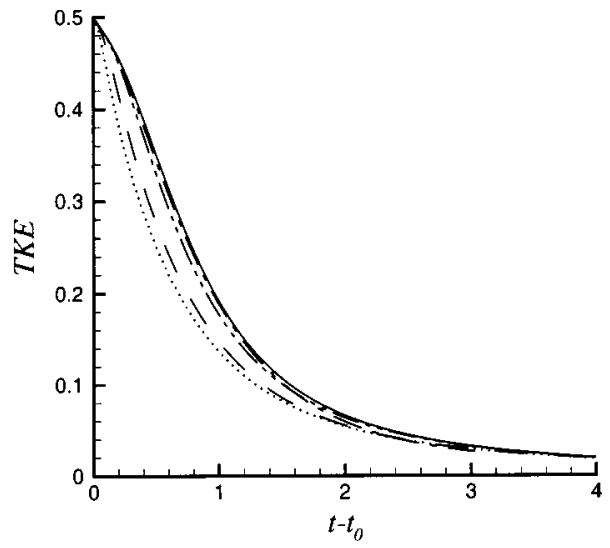

(b)

FIG. 20. The effect of the filter aspect ratio in the dynamic LES with the CBC initial data. (a) Dynamic LES $48^{3}$, (b) dynamic LES $64^{3}$. dynamic LES with a filter ratio 1.3 (dash); dynamic LES with a filter ratio 2 (dotted); dynamic LES with a filter ratio 4 (dash dot); full DNS $128^{3}$ (solid); DNS sharply filtered to $64^{3}$ (dash dot dot). 


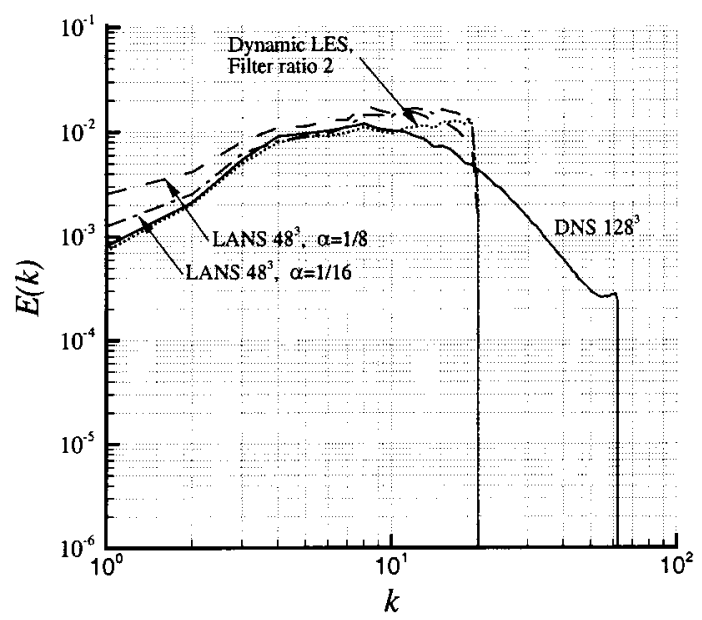

(a)

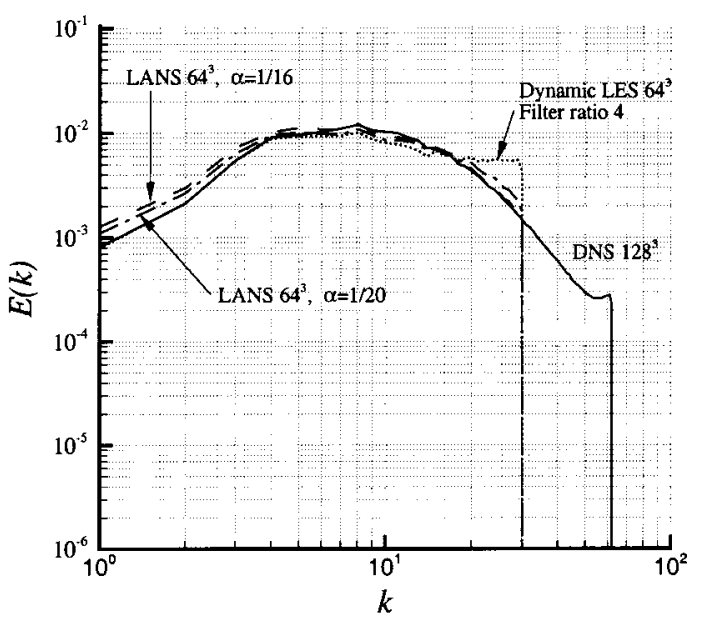

(b)

FIG. 21. The energy spectra at $t=1$ for the CBC initial data.

computations are presented. The pile-up of energy at higher wave numbers in the $48^{3}$ runs indicates insufficient dissipation of energy due to inadequate resolution. This is more pronounced in the LANS- $\alpha$ computations where the model is heavily dependent on the nonlinear energy redistribution mechanism of the Lagrangian-averaged equations as opposed to the dissipative model in the LES. For $64^{3}$ calculations the energy spectrum is predicted reasonably well by both the LANS- $\alpha$ and dynamic LES. The LANS- $\alpha$ computations show better agreement for higher wave numbers. At later times a dip at the peak of the energy spectrum is observed, which is more pronounced in the LANS- $\alpha$ simulations. This might be due to the introduction of the nonlinear energy redistribution effects in the LANS- $\alpha$ equations at scales of the order of $\alpha$. However, due to the broadband nature of the energy spectrum with the maximum of the spectrum at a relatively high wave number, we could not move $\alpha$ far from the energy-containing range. It is expected that in a higher Reynolds number flow, where there is an

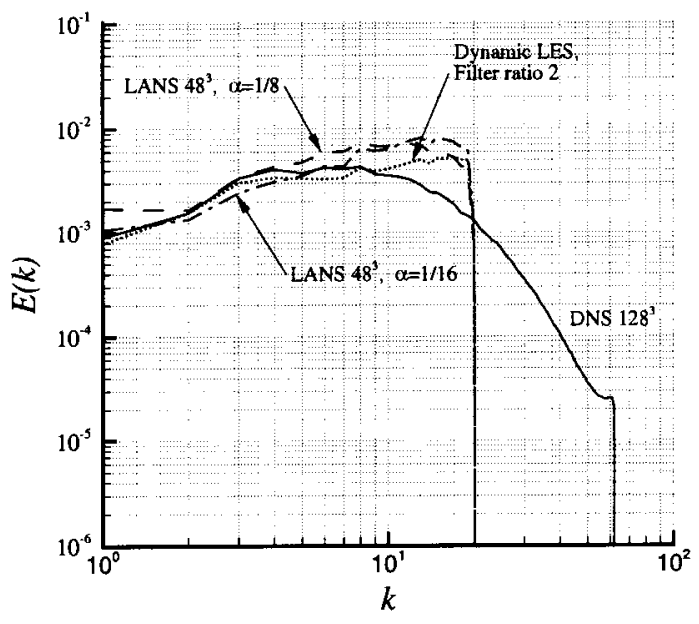

(a) extended inertial range and therefore a larger gap between the energy-containing scales and the $\alpha$ scale, this effect will be diminished. Existence of such a clearance between $\alpha$ scales and the location of the energy peak requires resolving at least a portion of the inertial subrange. LES methods will of course also work better in such a situation.

\section{B. Second decaying turbulence case}

In this section we perform a DNS computation of a decaying isotropic homogeneous turbulence with an energy spectrum peaked at the wave number 3 , and the resolution of the DNS computation is increased to $170^{3}\left(256^{3}\right.$ before dealiasing).

We start with a divergence free velocity field with a specified energy spectrum given by

$$
E(k)=A k^{4} \exp ^{-2 k^{2} / k_{0}^{2}},
$$

where $k$ is the wave number, $k_{0}$ is the wave number of the

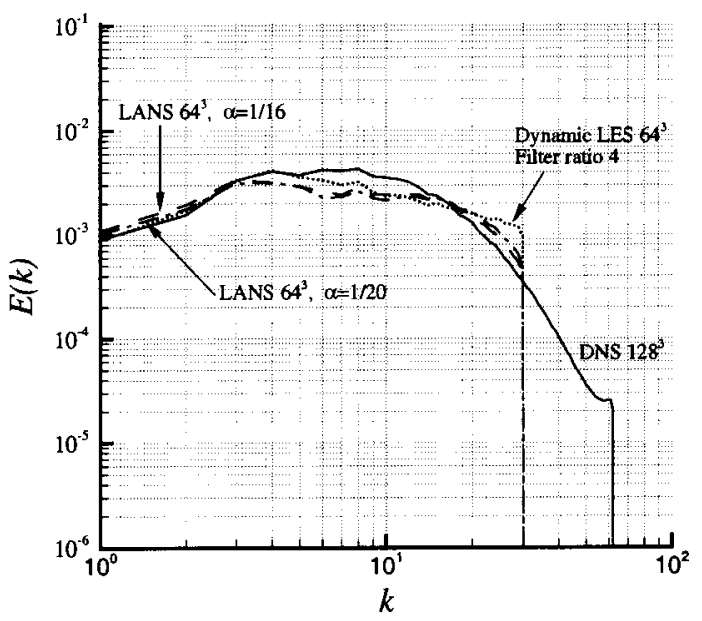

(b)

FIG. 22. The energy spectra at $t=2$ for the CBC initial data. 


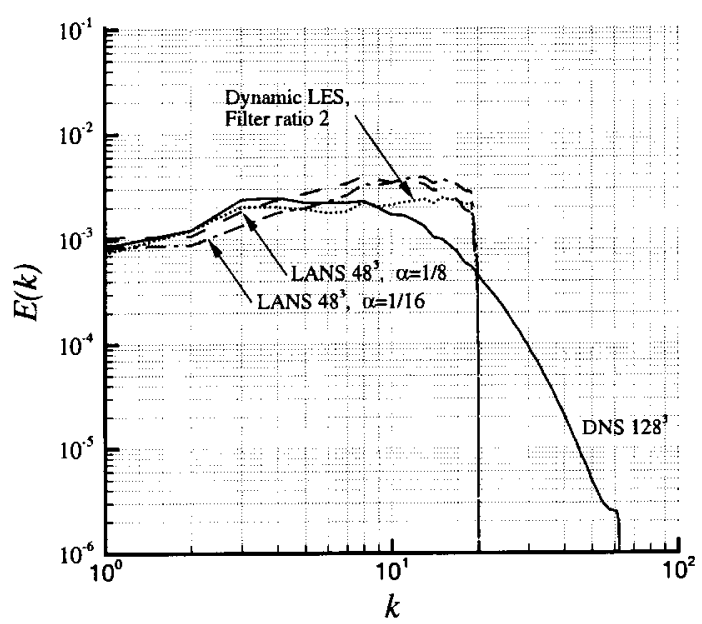

(a)

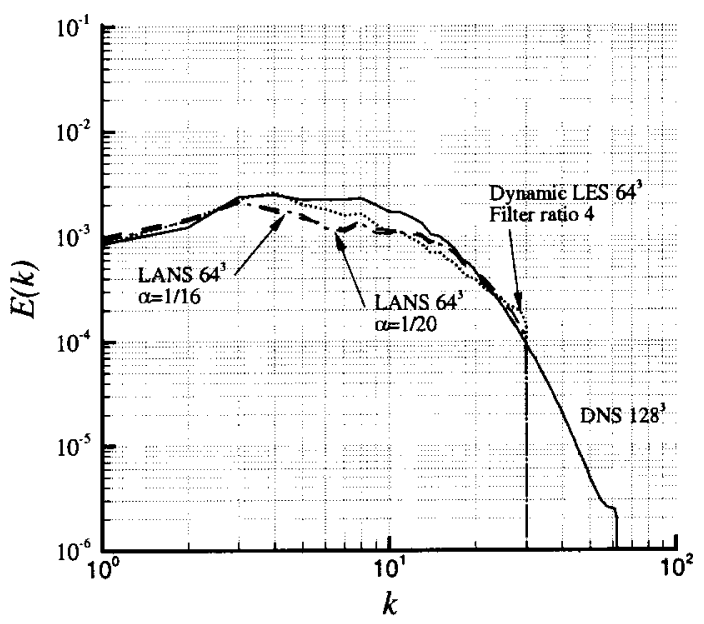

(b)

FIG. 23. The energy spectra at $t=3$ for the CBC initial data.

peak of the energy spectrum, and $A$ is a normalizing factor. In all of the computations presented in this study we use $k_{0}$ $=3$, and we choose $A$ such that the total kinetic energy of the initial velocity field is 0.5 . The initial Taylor Reynolds number is $\operatorname{Re}_{\lambda}=220$.

The evolution of the energy spectrum in the DNS using $170^{3}$ points is illustrated in Fig. 24. The energy of fully developed isotropic turbulence decays in time while the scales of motion grow. As a result of the total kinetic energy (TKE) decay the resulting $\operatorname{Re}_{\lambda}$ decreases with time. Consequently, a well resolved, fully developed field will remain well resolved as the TKE decays.

In Fig. 25 the evolution of the TKE of the DNS data is contrasted against various dynamic LES and the LANS- $\alpha$ simulations, for two resolutions: $48^{3}$ and $64^{3}$. TKEs for the DNS data, sharply filtered to the resolution of the LES and the LANS- $\alpha$ computations, are also presented. Dynamic LES

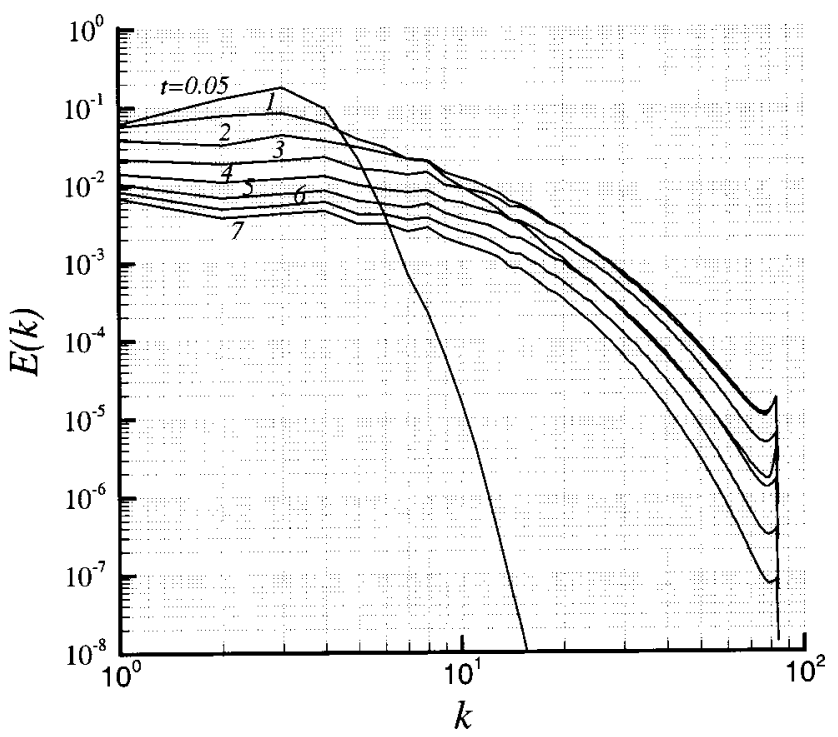

FIG. 24. Energy spectrum for the second decay case, $\mathrm{Re}_{\lambda}=220, N_{\mathrm{DNS}}^{3}$ $=170^{3}$. computations for filter aspect ratios of $1.11,1.33,2$, and 4 are performed. We found that the best match between the DNS data and the dynamic LES results is achieved for a filter aspect ratio of 1.11 for both $48^{3}$ and $64^{3}$ calculations (see Fig. 26). In both the $48^{3}$ and $64^{3}$ computations the LES and the LANS- $\alpha$ satisfactorily predict the decay rate. This should be contrasted with the results for the simulation of the CBC experiment in Sec. VI A where a low resolution calculation at $48^{3}$ was significantly in error. This confirms the speculation that for an accurate calculation based on the LANS- $\alpha$ or LES the bulk part of the energy containing wave modes should be included in the simulation. The same conclusion is drawn from Figs. 27 and 28 where the evolution of the energy spectrum of various computations are presented. All $48^{3}$ simulations indicate insufficient dissipation of energy at later times due to inadequate resolution. This is more pronounced in the LANS- $\alpha$ computations with $\alpha=1 / 16$ where the model is heavily dependent on the nonlinear energy redistribution mechanism of the Lagrangian-averaged equations (activated at a higher wave number) as opposed to the dissipative model in LES. For $64^{3}$ calculations the energy spectrum is predicted reasonably well by both the LANS- $\alpha$ and the dynamic LES.

\section{CONCLUSIONS}

Our objective in this study was to investigate the utility of the Lagrangian averaged Navier-Stokes (the LANS- $\alpha$ ) equations as a subgrid scale model for three-dimensional isotropic forced and decaying turbulence. Both viscous and inviscid computations were carried out. The essence of the Lagrangian averaging method is the nonlinear redistribution (not dissipative) nature of the energy decay in the Lagrangian averaged Euler (LAE- $\alpha$ ) equations, where the energy is removed from the small scales while maintaining the crucial features of the large scale flow. The final $L_{2}(u)$ level depends on the spectrum of the initial field, the size of the computational box, and $\alpha$. In the viscous counterpart (the LANS- $\alpha$ equations) the nonlinear redistributing energy de- 


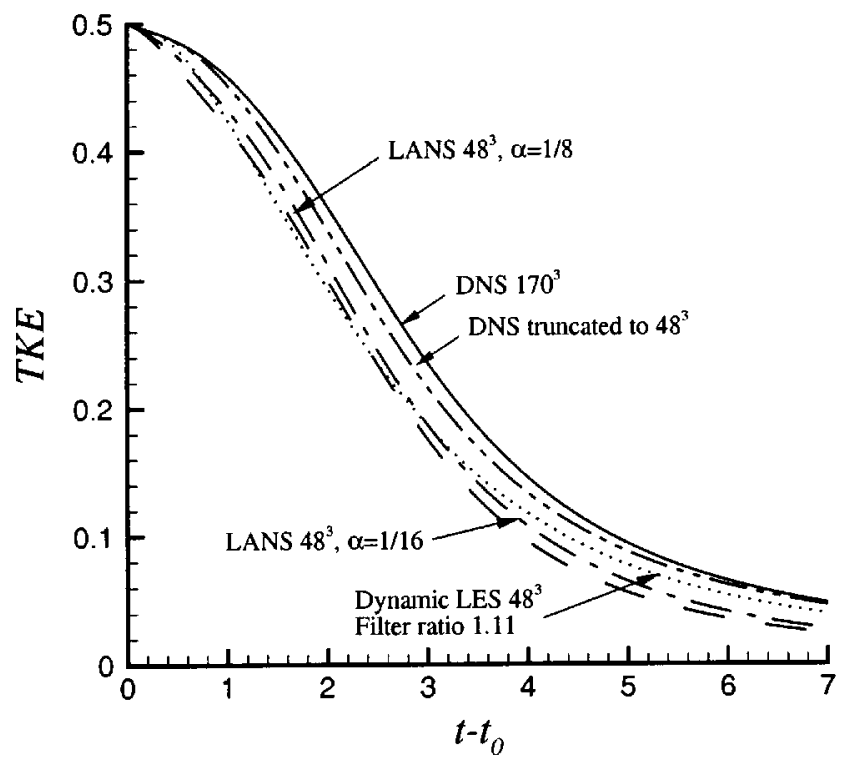

(a)

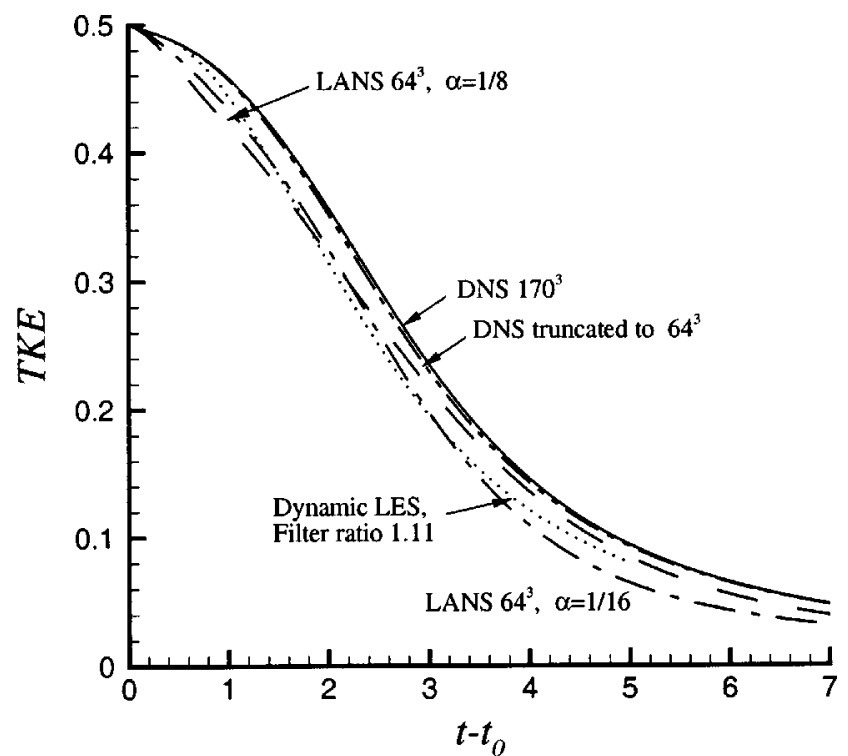

(b)

FIG. 25. Decay of kinetic energy for the second decay case; (a) $N_{\alpha}^{3}=48^{3}$; (b) $N_{\alpha}^{3}=64^{3}$. The filter aspect ratio in the dynamic LES is an extra free parameter. The dynamic LES computations were repeated for many filter aspect ratios (at least four), and only the results for the filter ratio with the best match with the DNS data are reported here (see also Fig. 26).

cay is accompanied by a viscous dissipation. Therefore, the LANS- $\alpha$ equations can be considered as a nonlinearly energy redistributive modification of the Navier-Stokes equations. This modification appears in the nonlinearity, depends on the scale $\alpha$, limits the effect of vortex stretching, and causes the energy spectrum to fall rapidly for scales smaller than $\alpha$. To determine the slope of this drop at higher wave modes one can in principle use an argument similar to Kolmogorov's argument which gives $-5 / 3$ inertial range slope. Results of various simulations of the LANS- $\alpha$ equations are compared with the dynamic large eddy simulations (LES) and fully

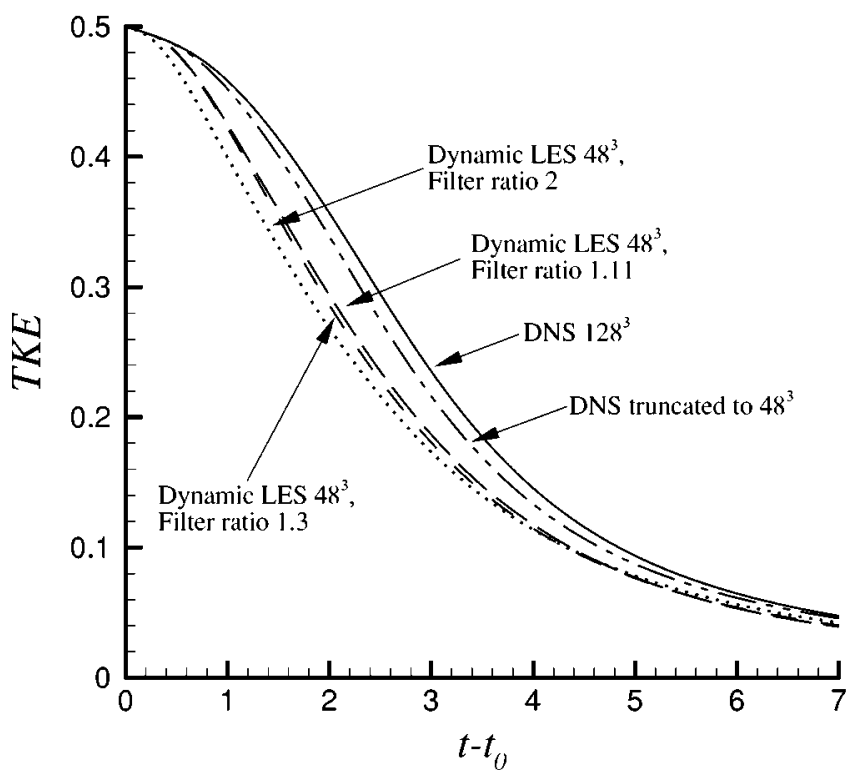

resolved direct numerical simulations (DNS). The filter aspect ratio in the dynamic LES model is a free parameter and the final result depends on the value of this parameter particularly in severe test cases such as the one considered here. In order to avoid introducing any further arbitrary parameters, the LES computations were repeated for many filter aspect ratios (at least four), and the parameter that matched the best with the turbulence decay of the DNS data or the DNS energy spectra was used for comparison with the LANS- $\alpha$ equations.

We performed two sets of forced isotropic turbulence

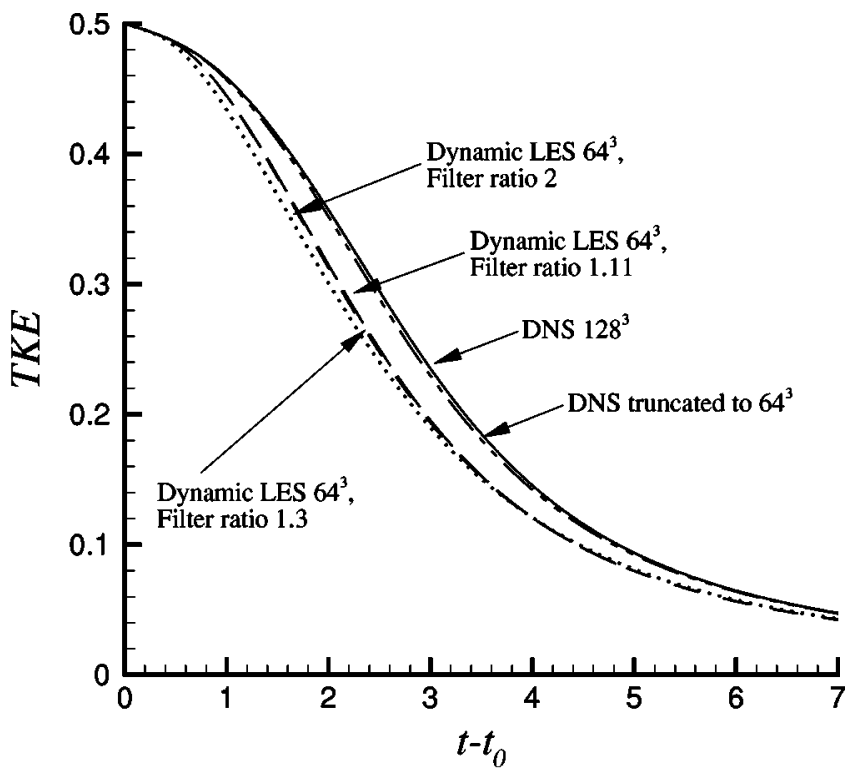

FIG. 26. The effect of the filter aspect ratio in the dynamic LES with the second initial data depicted in Fig. 24. (a) Dynamic LES $48^{3}$, (b) dynamic LES $64^{3}$. Dynamic LES with a filter ratio 1.11 (long dash); dynamic LES with a filter ratio 1.3 (dash); dynamic LES with a filter ratio 2 (dotted); full DNS $128^{3}$ (solid); DNS sharply filtered to $64^{3}$ (dash dot dot). 


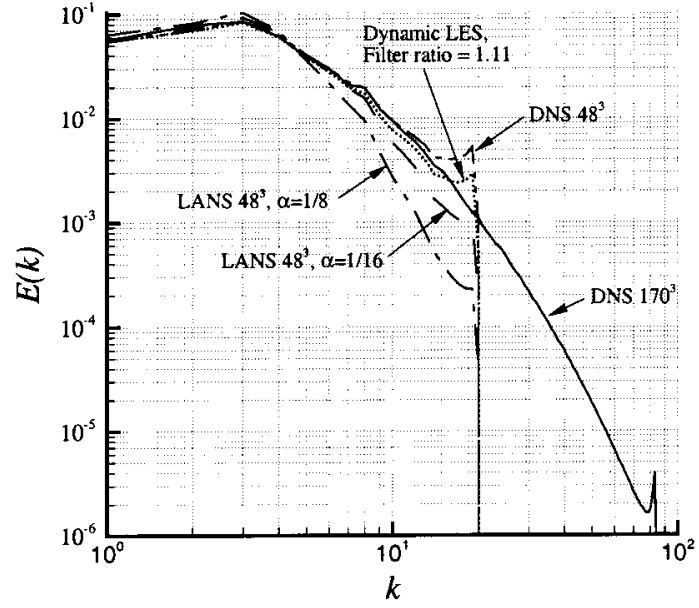

(a)

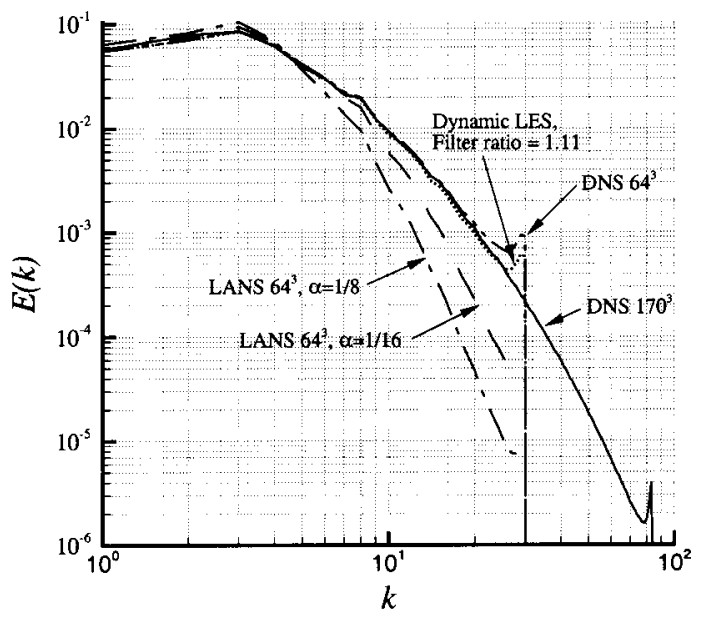

(b)

FIG. 27. The energy spectra at $t=1$ for the second decay case; (a) $N_{\alpha}^{3}=48^{3}$, (b) $N_{\alpha}^{3}=64^{3}$.

simulations of the LANS- $\alpha$ equations and studied their equilibrium states. The results of the LANS- $\alpha$ simulations were compared to the results obtained from high-resolution DNS simulations, characterized by the equilibrium Taylor Reynolds numbers $\operatorname{Re}_{\lambda}=80$ and 115 for two different forcing schemes. Furthermore, we studied the effect of varying the model scale $\alpha$. In general, selecting an appropriate $\alpha$ is a compromise between the accuracy at large scales and the minimum resolution requirement in the LANS- $\alpha$ simulations. The LANS- $\alpha$ equations accurately mimic the behavior of the Navier-Stokes equations at small wave numbers (large scales) as long as a minimum resolution is observed. At the wave number $k_{\alpha} \approx 1 / \alpha$ the slope of the spectra changes from the Navier-Stokes spectra to a much steeper slope. The smaller the $\alpha$ the larger the overlapping region of the low wave number spectra of the LANS- $\alpha$ and the Navier-Stokes equations. However, this higher accuracy comes with a price: The smaller $\alpha s$ require higher resolution for the LANS- $\alpha$ simulations. An underresolved LANS- $\alpha$ computation with the scale $\alpha$ close to the peak of the energy spectrum could result in the contamination of large scales and loss of accuracy.

The equilibrium energy spectrum in our LANS- $\alpha$ simulations were in good agreement with the DNS data at low wave numbers. Our simulations, in agreement with previous results by Chen et al. ${ }^{26}$ show dependency of the equilibrium turbulence spectra on the model scale $\alpha$. In the forced computations the slope of the energy spectra changes from the inertial range slope of $k^{-5 / 3}$ to a much steeper slope for scales smaller than $\alpha$. Even with $170^{3}$ grid-point LANS- $\alpha$ simulations, due to the insufficient resolution, we were not able to determine this second slope and confirm or falsify its existing estimates (e.g., Foias et al. ${ }^{39}$ ). We analyzed the energy transfer and the capability of the LANS- $\alpha$ in representing the backscatter of energy by comparison with the DNS data. We found that the LANS- $\alpha$ simulations accurately predict the transfer of energy to small scales. We have also tested the ability of the LANS- $\alpha$ equations to reproduce the

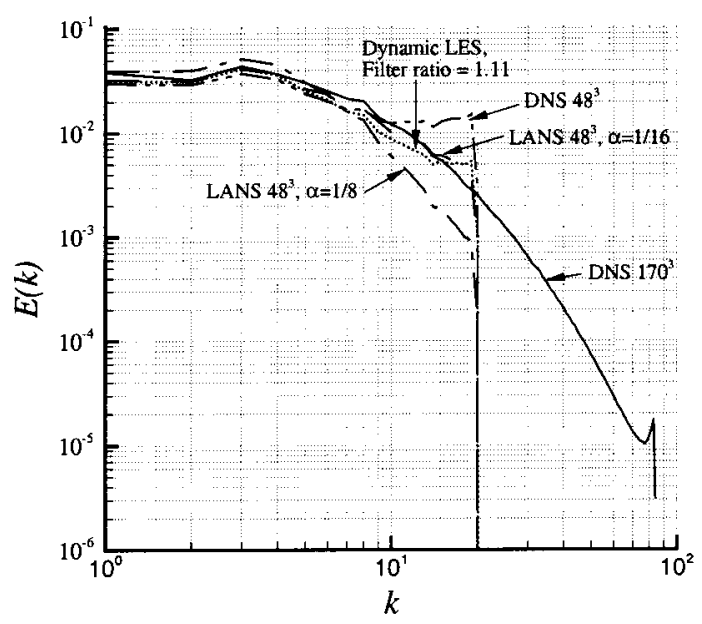

(a)

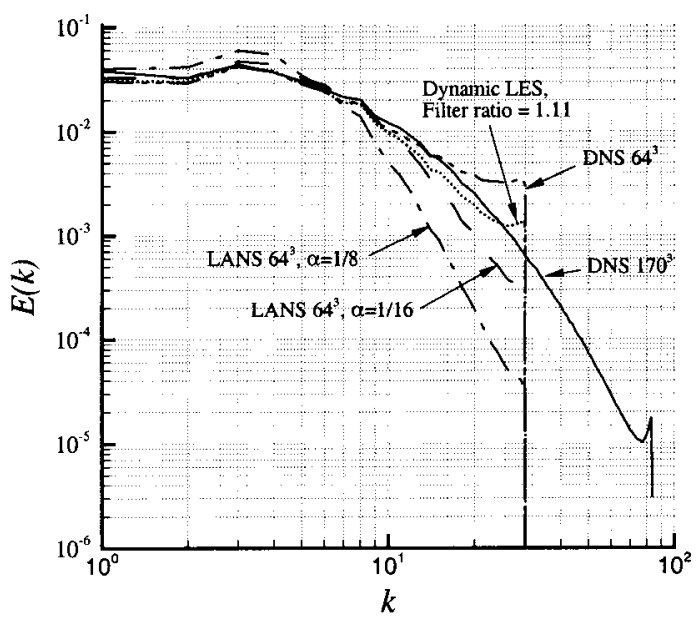

(b)

FIG. 28. The energy spectra at $t=2$ for the second decay case; (a) $N_{\alpha}^{3}=48^{3}$, (b) $N_{\alpha}^{3}=64^{3}$. 
observed alignments of eigenvectors of the subgrid stresses with respect to eigenvectors of the resolved stress tensor and vorticity vector. The LANS- $\alpha$ qualitatively reproduce the observed alignments in experiments and DNS data. In this respect the LANS- $\alpha$ equations are superior to linear turbulence models (including the dynamic LES) which imply perfect alignment between eigenvectors of the subgrid stress and the resolved strain rate tensor. The average relative angle between the most compressive eigendirection of the resolved strain rate tensor and the most extensive eigendirection of the subgrid stress tensor is captured accurately by the LANS- $\alpha$ equations.

Our conclusions for the decaying turbulence are made based on two separate numerical simulations. In the first case we start with a broad initial energy spectrum at $\operatorname{Re}_{\lambda}=72$ (CBC initial spectrum) where the peak of the energy spectrum is around the eighth Fourier mode. Correct prediction of the TKE decay rate and the corresponding spectra for such a broadband initial spectrum represents a difficult test case for any turbulence model. In the second decaying case we performed direct numerical simulation of the Navier-Stokes equations at a Taylor Reynolds number of $\operatorname{Re}_{\lambda}=220$ in a periodic box. The initial energy spectrum was peaked at a relatively low wave number (third Fourier mode), making it a suitable test case for testing the LANS- $\alpha$ modeling capabilities.

We found that, if a minimum resolution requirement (for the first decaying experiment at $\operatorname{Re}_{\lambda}=72$ half of the DNS resolution) is satisfied, then the LANS- $\alpha$ equations provide a satisfactory turbulence closure comparable with the dynamic LES. This minimum resolution depends on the initial energy spectrum. The only free parameter in the LANS- $\alpha$ simulations is a scale $\alpha$ which is representative of the spatial scale of the Lagrangian averaging. However, for lower resolutions both the LANS- $\alpha$ and dynamic LES show a pile-up of energy at higher wave numbers. This indicates insufficient dissipation of energy due to the lack of resolution.

Any conclusion on the performance of the LANS- $\alpha$ equations depends strongly on the way that the DNS data are compared with the results of the LANS- $\alpha$ calculations. First of all, appropriate interpretation of the Lagrangian averaging process of the DNS data is crucial for obtaining the initial velocity field for the LANS- $\alpha$ computations. The same issue arises in comparing the LANS- $\alpha$ results with DNS data at later times. We considered a few different approaches. Apart from truncation of the DNS data to the resolution of the LANS- $\alpha$ simulations, we have initialized the LANS- $\alpha$ simulations by Helmholtz filtering or spatially averaging of the DNS data. For initial energy spectrum with sharp peaks at low wave numbers and a value of $\alpha$ sufficiently away from this peak, one expects that the Helmholtz filtering of the initial data will not have a significant effect on the resolved TKE. However, for the very broad initial energy spectrum of the $\mathrm{CBC}$ experiment we found that using a filter based on the Helmholtz operator or spatial averaging resulted in a severe reduction of the initial resolved kinetic energy to a fraction of that of a field truncated in Fourier space (i.e., using a Fourier cut-off filter). We point out that such a reduction is independent of the model used and diminishes the value of the tests. We therefore use sharply truncated DNS data as the initial velocity field $(u)$ in the LANS- $\alpha$ simulations.

Recently, Chen et al. ${ }^{40}$ simulated a forced homogeneous turbulent flow and reported favorable results using the LANS- $\alpha$ equations. Here, we demonstrated the modeling capabilities of the LANS- $\alpha$ equations in decaying turbulent flows. Apart from these satisfactory results the LANS- $\alpha$ equations have many attractive theoretical features that make them a promising candidate for more complicated problems. In this process the next natural step is to test the LANS- $\alpha$ model in anisotropic flows. Numerical simulation of channel flow based on the anisotropic LANS- $\alpha$ equations is the topic of our future research.

In summary we conclude that for the test cases considered in this study the LANS- $\alpha$ equations can capture most of the large scale features of the turbulent flow while the effect of small scales on the large scales were modeled by Lagrangian averaging.

\section{ACKNOWLEDGMENTS}

The authors would like to thank the Center for Turbulence Research (CTR) for the opportunity to visit the Center during the summer program 2000. We would also like to thank P. Moin, A. Wray, R. Rogallo, and D. Carati, for their help and useful comments during our stay at the CTR summer program 2000. The data for the initial condition for the DNS computations in Sec. VI A was provided by A. Wray. Most of the simulations were run while K.M. was at the California Institute of Technology. Work was partially supported by the NSF-KDI Grant No. ATM-98-73133. J.E.M. also acknowledges the support of the California Institute of Technology and S.S. was partially supported by the Alfred P. Sloan Foundation Research Fellowship, and IGPP Los Alamos National Laboratory minigrant, and the NSF Grant No. DMS-0105004. K.M. and J.E.M. received partial support from the AFOSR, Grant No. 1046925.

${ }^{1}$ R. S. Rogallo and P. Moin, "Numerical simulations of turbulent flows," Annu. Rev. Fluid Mech. 16, 99 (1984).

${ }^{2}$ C. Canuto, M. Y. Hussaini, A. Quarteroni, and T. A. Zang, Spectral Methods in Fluid Dynamics, Springer Series in Computational Physics (Springer-Verlag, Berlin, 1988).

${ }^{3}$ D. K. Lilly, "The representation of small-scale turbulence in numerical experiment," in Proceedings of IBM Scientific Computing Symposium on Environmental Sciences (IBM, White Plains, NY, 1967), pp. 195-210.

${ }^{4}$ J. Smagorinsky, "General circulation experiments with the primitive equations," Month. Wea. Rev. 91, 99 (1963).

${ }^{5}$ J. W. Deardorff, "A numerical study of three-dimensional turbulent channel flow at large Reynolds numbers," J. Fluid Mech. 41, 453 (1970).

${ }^{6} \mathrm{U}$. Schumann, "Subgrid scale model for finite difference simulation of turbulent flows in plane channels and annuli," J. Comput. Phys. 18, 376 (1975).

${ }^{7}$ A. Leonard, "Energy cascade in large-eddy simulations of turbulent fluid flows," Adv. Geophys. 18, 237 (1974).

${ }^{8}$ O. J. McMillan and J. H. Ferziger, "Direct testing of subgrid-scale models," AIAA J. 17, 1340 (1979).

${ }^{9}$ W. C. Reynolds, R. A. Clark, and J. H. Ferziger, "Evaluation of subgridscale models using an accurately simulated turbulent flow," J. Fluid Mech. 91, 1 (1979).

${ }^{10}$ J. Bardina, J. H. Ferziger, and W. C. Reynolds, "Improved turbulence models based on large eddy simulation of homogeneous, incompressible turbulent flows," Report TF-19, Thermoscience Div., Department of Mechanical Engineering, Stanford University, Stanford, CA, 1983.

${ }^{11}$ C. Meneveau and J. Katz, "Scale-invariance and turbulence models for 
large-eddy simulation,” Annu. Rev. Fluid Mech. 32, 1 (2000).

${ }^{12}$ M. Germano, U. Piomelli, P. Moin, and W. H. Cabot, "A dynamic subgrid scale eddy viscosity model," Phys. Fluids A 3, 1760 (1991).

${ }^{13}$ R. S. Rivlin, "The relation between the flow of non-Newtonian fluids and turbulent Newtonian flows," Q. Appl. Math. 15, 212 (1957).

${ }^{14}$ P. J. Mason, "Large-eddy simulation: A critical review of the technique," Q. J. R. Meteorol. Soc. 120, 1 (1994).

${ }^{15}$ M. Lesieur and O. Métais, "New trends in large-eddy simulation," Annu. Rev. Fluid Mech. 28, 45 (1996).

${ }^{16}$ T. J. R. Hughes, L. Mazzei, and K. E. Jansen, "Large eddy simulation and the variational multiscale method," Computing and Visualization in Science 3, 47 (2000).

${ }^{17}$ T. J. R. Hughes, L. Mazzei, A. A. Oberai, and A. A. Wray, "The multiscale formulation of large eddy simulation: Decay of homogeneous isotropic turbulence," Phys. Fluids 13, 505 (2001).

${ }^{18}$ T. J. R. Hughes, A. A. Oberai, and L. Mazzei, "Large eddy simulation of turbulent channel flows by the variational multiscale method," Phys. Fluids 13, 1784 (2001)

${ }^{19}$ S. S. Collis, "Monitoring unresolved scales in multiscale turbulence modeling," Phys. Fluids 13, 1800 (2001).

${ }^{20}$ D. D. Holm, J. E. Marsden, and T. S. Ratiu, "Euler-Poincaré models of ideal fluids with nonlinear dispersion,” Phys. Rev. Lett. 80, 4173 (1998).

${ }^{21}$ J. E. Marsden and S. Shkoller, "Global well-posedness for the Lagrangian averaged Navier-Stokes (LANS- $\alpha$ ) equations on bounded domains," Proc. R. Soc. London 359, 1449 (2001).

${ }^{22}$ D. D. Holm, J. E. Marsden, and T. S. Ratiu, "The Euler-Poincaré equations and semidirect products with applications to continuum theories," Adv. Math. 137, 1 (1998).

${ }^{23}$ S. Shkoller, "Geometry and curvature of diffeomorphism groups with $H^{1}$ metric and mean hydrodynamics," J. Funct. Anal. 160, 337 (1998).

${ }^{24}$ J. E. Marsden, T. Ratiu, and S. Shkoller, "The geometry and analysis of the averaged Euler equations and a new diffeomorphism group," Geom. Funct. Anal. 10, 582 (2000).

${ }^{25}$ S. Shkoller, "Analysis on groups of diffeomorphisms of manifolds with boundary and the averaged motion of a fluid," J. Diff. Geom. 55, 145 (2000).

${ }^{26}$ S. Y. Chen, D. D. Holm, L. G. Margoin, and R. Zhang, "Direct numerical simulations of the Navier-Stokes alpha model," Physica D 133, 66 (1999).

${ }^{27}$ K. Mohseni, S. Shkoller, B. Kosovic, and J. Marsden, "Numerical simulations of forced homogeneous turbulence using the Lagrangian averaged Navier-Stokes equations," AIAA Paper 2001-2645, 2001.

${ }^{28} \mathrm{G}$. Comte-Bellot and S. Corrsin, "The use of a contraction to improve the isotropy of grid-generated turbulence," J. Fluid Mech. 25, 657 (1966).

${ }^{29}$ G. Comte-Bellot and S. Corrsin, "Simple Eulerian time correlation of full and narrow-band velocity signals in grid-generated isotropic turbulence," J. Fluid Mech. 48, 273 (1971).

${ }^{30}$ K. Mohseni, S. Shkoller, B. Kosovic, J. Marsden, D. Carati, A. Wray, and R. Rogallo, "Numerical simulations of homogeneous turbulence using Lagrangian averaged Navier-Stokes equations," in Proceedings of the 2000 Summer Program, NASA Ames/Stanford University, 2000, pp. 271283

${ }^{31}$ J. E. Marsden and S. Shkoller, "The anisotropic averaged Euler equations," Arch. Ration. Mech. Anal. (to be published).

${ }^{32}$ C. Foias, D. D. Holm, and E. S. Titi, "The three-dimensional viscous Camassa-Holm equations and their relation to the Navier-Stokes equations and turbulence theory," J. Dyn. Diff. Eq. 14, 1 (2002).

${ }^{33}$ M. Oliver and S. Shkoller, "The vortex method as a second-grade nonNewtonian fluid," Commun. Partial Differ. Equ. 26, 91 (2001).

${ }^{34}$ S. Shkoller, "Smooth global Lagrangian flow for the 2D Euler and second-grade fluid equations," Appl. Math. Lett. 14, 539 (2001).

${ }^{35}$ A. Chorin, "Numerical study of slightly viscous flow," J. Fluid Mech. 57, 785 (1973)

${ }^{36}$ C. S. Peskin, "A random-walk interpretation of the incompressible Navier-Stokes equations,” Eur. Phys. J. A 38, 845 (1985).

${ }^{37}$ G. I. Taylor, "The spectrum of turbulence," Proc. R. Soc. London, Ser. A 164, 476 (1938).

${ }^{38}$ S. Y. Chen, C. Foias, D. D. Holm, E. Olson, E. S. Titi, and S. Wynne, "The Camassa-Holm equations as a closure model for turbulent channel and pipe flow," Phys. Rev. Lett. 81, 5338 (1998).

${ }^{39}$ C. Foias, D. D. Holm, and E. S. Titi, "The Navier-Stokes- $\alpha$ model of fluid turbulence," Physica D 152, 505 (2001).

${ }^{40}$ S. Y. Chen, C. Foias, D. D. Holm, E. Olson, E. S. Titi, and S. Wynne,
"The Camassa-Holm equations and turbulence," Physica D 133, 49 (1999).

${ }^{41}$ S. Y. Chen, C. Foias, D. D. Holm, E. Olson, E. S. Titi, and S. Wynne, "A connection between the Camassa-Holm equations and turbulent flows in channels and pipes," Phys. Fluids 11, 2343 (1999).

${ }^{42}$ J. L. Lumley, "Toward a turbulent constitutive relation," J. Fluid Mech. 41, 413 (1970).

${ }^{43}$ S. B. Pope, "A more general effective-viscosity hypothesis," J. Fluid Mech. 72, 331 (1975).

${ }^{44}$ C. G. Speziale, "On nonlinear $k-l$ and $k-\epsilon$ models of turbulence," J. Fluid Mech. 178, 459 (1987).

${ }^{45}$ C. G. Speziale, "Analytical methods for the development of Reynoldsstress closures in turbulence," Annu. Rev. Fluid Mech. 23, 107 (1991).

${ }^{46} \mathrm{~A}$. Yoshizava, "Statistical analysis of the deviation of the Reynolds stress from its eddy-viscosity representation," Phys. Fluids 27, 1377 (1984).

${ }^{47}$ R. Rubinstein and J. M. Barton, "Nonlinear Reynolds stress models and the renormalization group," Phys. Fluids A 2, 1472 (1990).

${ }^{48}$ A. Leonard and G. S. Winckelmans, "A tensor-diffusivity subgrid model for large-eddy simulation," in Proceedings of the Isaac Newton Institute Symposium/ERCOFTAC Workshop, Direct and Large-eddy Simulations III, edited by P. R. Voke, N. D. Sandham, and L. Kleiser, Cambridge, U.K., May 12-14, 1999 (Kluwer Academic, New York, 1999), pp. 147162.

${ }^{49}$ S. Stolz, N. A. Adams, and L. Kleiser, "An approximate deconvolution model for large-eddy simulation with application to incompressible wallbounded flows," Phys. Fluids 13, 997 (2001).

${ }^{50}$ T. S. Lund and E. A. Novikov, "Parameterization of subgrid-scale stress by the velocity gradient tensor," in Annual Research Briefs, pp. 27-43, Stanford, CA, 1992.

${ }^{51}$ V. C. Wong, "A proposed statistical-dynamic closure method for the linear or nonlinear subgrid-scale stresses," Phys. Fluids A 4, 1080 (1992).

${ }^{52} \mathrm{~B}$. Kosović, "Subgrid-scale modelling for the large-eddy simulation of high-Reynold-number boundary layers," J. Fluid Mech. 336, 151 (1997).

${ }^{53}$ B. Kosović and J. A. Curry, "A large eddy simulation study of a quasisteady, stably stratified atmospheric boundary layer," J. Atmos. Sci. 57, 1052 (2000).

${ }^{54}$ D. Coutand and S. Shkoller, "Turbulent channel flow in weighted Sobolev spaces using the anisotropic Lagrangian averaged Navier-Stokes (LANS- $\alpha$ ) equations," preprint, 2002.

${ }^{55}$ S. A. Orszag and G. S. Patterson, "Numerical simulation of turbulence," Statistical Models and Turbulence, Volume 12 of Lecture Notes in Physics (Springer, New York, 1972).

${ }^{56}$ R. S. Rogallo, "Numerical experiments in homogeneous turbulence," in NASA Tech. Memo 81315, 1981.

${ }^{57}$ G. K. Batchelor, The Theory of Homogeneous Turbulence (Cambridge University Press, Cambridge, 1953).

${ }^{58} \mathrm{~A}$. N. Kolmogorov, "The local structure of turbulence in incompressible viscous fluids at very large Reynolds numbers," Dokl. Akad. Nauk SSSR 30, 301 (1941).

${ }^{59}$ E. D. Siggia and G. S. Patterson, "Intermittency effects in a numerical simulation of stationary three-dimensional turbulence," J. Fluid Mech. 86, 567 (1978).

${ }^{60}$ V. Eswaran and S. B. Pope, "An examination of forcing in direct numerical simulations of turbulence," Comput. Fluids 16, 257 (1988).

${ }^{61}$ S. Ghosal, T. S. Lund, P. Moin, and K. Akselvoll, "A dynamic localization model for large-eddy simulation of turbulent flows," J. Fluid Mech. 285, 229 (1995)

${ }^{62}$ D. Carati, S. Ghosal, and P. Moin, "On the representation of backscatter in dynamic localization models," Phys. Fluids 7, 606 (1995).

${ }^{63}$ A. Misra and D. I. Pullin, "A vortex-based subgrid stress model for largeeddy simulation,” Phys. Fluids 9, 2443 (1997).

${ }^{64} \mathrm{~S}$. Chen and X. Shan, "High-resolution turbulent simulations using the connection machine-2," Comput. Phys. 6, 643 (1992).

${ }^{65} \mathrm{U}$. Piomelli, P. Moin, and J. Ferziger, "Model consistency in large eddy simulation of turbulent channel flows," Phys. Fluids 31, 1884 (1988).

${ }^{66}$ Z. S. She, E. Jackson, and S. A. Orszag, "Intermittent vortex structures in homogeneous isotropic turbulence," Nature (London) 344, 226 (1990).

${ }^{67}$ A. Vincent and M. Meneguzzi, "The spatial structure and statistical properties of homogeneous turbulence," J. Fluid Mech. 255, 1 (1991).

${ }^{68}$ J. Jimenez, A. A. Wray, P. G. Saffman, and R. S. Rogallo, "The structure of intense vorticity in isotropic turbulence," J. Fluid Mech. 255, 65 (1993).

${ }^{69}$ T. Miyauchi and M. Tanahashi, "Coherent fine scale structure in turbulence," in Proceedings of the IUTAM Symposium on Geometry and Sta- 
tistics of Turbulence, Hayama (Kanagawa-ken), Japan, November 1-5, 1999, edited by T. Kambe, T. Nakano, and T. Miyauchi (Kluwer Academic, New York, 2001), pp. 67-76.

${ }^{70} \mathrm{~T}$. Miyauchi and M. Tanahashi, "Coherent fine scale eddied in turbulent shear flows and its relation to anisotropy," in Proceedings of the IUTAM Symposium on Geometry and Statistics of Turbulence, edited by T. Kambe, T. Nakano, and T. Miyauchi, Hayama (Kanagawa-ken), Japan, November 1-5, 1999 (Kluwer Academic, New York, 2001), pp. 77-82.

${ }^{71}$ B. Tao, J. Katz, and C. Meneveau, "Application of HPIV data of turbulent duct flow for turbulence modeling," in Proceedings of 3rd ASME/JSME Joint Fluids Engineering Conference, number FEDSM 99-7281, ASME, June 18-22 1999.

${ }^{72}$ B. Tao, J. Katz, and C. Meneveau, "Effects of strain-rate and subgrid dissipation rate on alignment trends between large and small scales in turbulent duct flow," in Proceedings of 3rd ASME Fluids Engineering Division Summer Meeting, number FEDSM 00-11164, ASME, June 1115,2000 .
${ }^{73}$ B. Tao, J. Katz, and C. Meneveau, "Geometry and scale relationships in high Reynolds number turbulence determined from three-dimensional holographic velocimetry," Phys. Fluids 12, 941 (2000).

${ }^{74}$ R. M. Kerr, "Higher-order derivative correlations and the alignment of small-scale structures in isotropic numerical turbulence," J. Fluid Mech. 153, 31 (1985).

${ }^{75}$ W. T. Ashurst, A. R. Kerstein, R. M. Kerr, and C. H. Gibson, "Alignment of vorticity and scalar-gradient in simulated Navier-Stokes turbulence," Phys. Fluids 30, 2343 (1987).

${ }^{76}$ G. I. Taylor, "Statistical theory of turbulence," Proc. R. Soc. London, Ser. A 151, 421 (1935)

${ }^{77}$ S. R. Stalp, L. Skrbek, and R. J. Donnelly, "Decay of grid turbulence in a finite channel," Phys. Rev. Lett. 82, 4831 (1999).

${ }^{78}$ I. P. D. De Silva and H. J. S. Fernando, "Oscillating grids as a source of nearly isotropic turbulence," Phys. Fluids 6, 2455 (1994).

${ }^{79}$ A. A. Wray, "A selection of test cases for the validation of large-eddy simulations of turbulent flows," AGARD Advisory Report 345, 1998. 